LAURO AFONSO CÔRTES BOGNIOTTI

AVALIAÇÃO DO PEPTÍDEO NATRIURÉTICO CEREBRAL (BNP) PARA PREDIÇÃO DE MORTALIDADE A CURTO PRAZO APÓS O INFARTO AGUDO DO MIOCÁRDIO EM COMPARAÇÃO COM O ESCORE GRACE: SUBANÁLISE DO BRASÍLIA HEART STUDY

BRASÍLIA - DF, 2016 
UNIVERSIDADE DE BRASÍLIA - UnB

FACULDADE DE MEDICINA

PROGRAMA DE PÓS-GRADUAÇÃO EM CIÊNCIAS MÉDICAS

LAURO AFONSO CÔRTES BOGNIOTTI

\section{AVALIAÇÃO DO PEPTÍDEO NATRIURÉTICO CEREBRAL (BNP) PARA PREDIÇÃO DE MORTALIDADE A CURTO PRAZO APÓS O INFARTO AGUDO DO MIOCÁRDIO EM COMPARAÇÃO COM O ESCORE GRACE: SUBANÁLISE DO BRASÍLIA HEART STUDY}

Dissertação de Mestrado a ser apresentada ao Programa de Pós-Graduação em Ciências Médicas da Faculdade de Medicina da Universidade de Brasília como pré-requisito à obtenção do título de Mestre em Ciências Médicas.

Orientador: Prof. Dr. Andrei Carvalho Sposito Co-orientador: Dr. Luiz Sérgio Fernandes de Carvalho

BRASÍLIA - DF 2016 
À minha família, meu alicerce;

A meus professores, habilidosos escultores de minha mente;

A meus amigos, irmãos nascidos de outros pais; Ao misterioso criador de todas as coisas, por vezes denominado Deus. 


\section{AGRADECIMENTOS}

Este trabalho somente pôde se materializar devido aos incomensuráveis esforços das pessoas que compõe a "família" Coorte Brasília. Idealizada de forma pioneira pelo Professor Dr. Andrei Sposito, que desde o princípio não mediu esforços para torná-la um ninho de vasto aprendizado médico, científico e, sobretudo, humano, a Coorte de fato se tornou uma grande família. Foi graças ao empenho de todos os "filhos, netos e bisnetos" do grupo, do qual tenho a honra e o orgulho de fazer parte desde 2010, que tive a condição de vencer os desafios que se impuseram ao longo deste caminho que hoje trilho. Agradeço, portanto, a todos os integrantes da Coorte Brasília, em especial àqueles com os quais tive o prazer de conviver: aos professores, mestres e doutores Andrei Sposito, Luiz Sérgio Carvalho (meu co-orientador, grande amigo e exemplo, fundamental em todos os momentos deste trabalho), Osório Rangel, José Carlos Quinaglia, Otávio Rizzi, Wilson Nadruz, Maurício Daher, Thiago Quinaglia, Francisco Neves, Daniel Munhoz; aos "irmãos" da Coorte Tiago Morato, Augusto Gurgel, Antônio Carlos Macedo, Riobaldo Cintra, Ana Laura Araújo, André Hideo Ono, Alison Alexandre, Bruno Alvarenga, Uelver Cintra, Alexandre Anderson, André Japiassú, Luiza Pego, Guilherme de Carvalho, Filipe Moura, Juliana de Pina Araújo, Marco Antônio Freitas, Fernando Souza, Naiara Viudes, Rodrigo Lobo, Frank Venâncio, Lara Romero, Carolina Alves, Mário Nóbrega, Foncieli Fontana, Bruno Sakamoto, Nestor Martins, Meyrianne Almeida, Bruna Teles, Carla Borges, Lara Ribeiro, Jéssica Silvério, Breno Faria, Fillipe Frod, Gabriel Salmen, Luciana Trindade, Marcelo Artiaga, Rodrigo Barbosa, Gabriela Oliveira, Patrícia Monteiro, Pedro Beal, José Teófilo, Milena Adolfo, Armindo Jreige.

Naturalmente, agradeço a toda a minha família: a meus pais, por todo o apoio e carinho imprescindíveis nos momentos de calmaria ou turbulência; ao meu irmão Lucas, o qual admiro muito e pelo qual tenho muita estima, e que recentemente também ingressou na família Coorte; aos meus avós e tios, pelos momentos especiais de convivência e aprendizado.

Não posso me esquecer também da imensa colaboração de meus "irmãos de farda" do Exército Brasileiro e do Hospital das Forças Armadas, onde aprendi a disciplina e a união, e pude dar os primeiros passos dentro da profissão. Agradeço em especial à Comandante Stella Taylor, que tanto me incentivou e apoiou neste desafio, 
e aos meus amigos e colegas Leonardo Boggio, Naiara Galvão, João Ricarte, André Batista, Wigney Borges, Giovanny, Silval, José Gonçalves, Luis Danda, João Volpe, Eduardo Grisolia, com os quais passei algumas noites em claro e pude aprender muito neste início de jornada.

Agradeço aos todos os meus amigos, que sempre que podem me privilegiam com sua presença e excelentes conversas, em especial aos queridos João Costa Neto, Felipe Cavalcante, Matheus Scussel, Vitor Horita, Vagner Horita, Victor Bittar, Danilo Souza, Mariana Marques, Talyta Grippe, Bruno Luitgards, Leiliane Sampaio.

Por fim, agradeço a todos os pacientes do estudo, pelo seu generoso gesto de doação em um momento de dor (física e emocional), para que com eles pudéssemos aprender, na louvável tentativa de ajudar ao próximo que também sofre. 
"Doctors are men who prescribe medicines of which they know little, to cure diseases of which they know less, in human beings of whom they know nothing"

Voltaire, 1694 - 1778 


\section{RESUMO}

Introdução: Os escores clínicos disponíveis para a predição de risco após o infarto agudo do miocárdio (IAM) têm mostrado um potencial limitado para discriminar pacientes de alto risco. Embora a concentração plasmática de BNP na admissão possa predizer a probabilidade de eventos adversos após IAM, não há informações sobre a sua capacidade preditiva em pacientes com IAM e função ventricular normal na admissão (Killip I).

Objetivo: avaliar o BNP e o escore GRACE em pacientes com IAMCSST em uma coorte prospectiva, especificamente naqueles com Killip I à admissão hospitalar

Métodos: Pacientes consecutivos com IAM com supradesnivelamento do segmento ST e Killip I $(n=167)$ foram acompanhados por 12 meses. Foram avaliados os níveis plasmáticos de BNP nas primeiras 24 horas após o início dos sintomas (D1) e no $5^{\circ}$ dia (D5) e calculado o escore GRACE com base nos dados da admissão.

Resultados: Fatores de risco independentes foram selecionados usando o método bootstrapping com base em regressões de Cox. Sexo, idade, dose de estatina e diagnóstico de diabetes mellitus foram selecionadas como preditores significativos de morte súbita e IAM (MACE) para o modelo multivariado final. Tanto em modelos nãoajustados e ajustados de regressão de Cox, tanto um Delta-BNP acima da mediana (80pg/dL) e o BNP à admissão (D1) acima de 100pg/dL (mediana) foram associados a maior incidência de morte súbita e IAM em 30 dias (OR:10.88, 95\% Cl: 1.10-108, $\mathrm{p}=0.038$; OR:1.91, 95\%Cl: 1.01-4.11, $\mathrm{p}=0.049$, respectivamente). Escore GRACE isolado mostrou uma estatística- $C$ moderada de 0,709 ( $p=0,029)$, mas ao se associar o Delta-BNP houve melhora na discriminação do risco (estatística $\mathrm{C}=0,831, \mathrm{p}=0,001$ ), com diferenças significativas entre as áreas sob a curva $(p=0,046)$. A reclassificação liquida (category-free NRI) confirmou uma melhoria significativa na predição do risco em 33,4\% (IC 95\%: 8-61\%) em comparação com o GRACE isolado ( $p=0,034$ ), com calibração adequada de acordo com critério de informação de Bayes e teste da razão de verossimilhança. A associação entre Delta-BNP + GRACE melhorou a reclassificação especialmente em doentes de risco intermediário [em 62,5\% $(p=0,0231)]$. No entanto, considerando apenas a primeira medida, o BNP no D1 não melhorou a reclassificação de risco em 30 dias quando comparado ao escore GRACE 
(cfNRI $p=0.8$ ), assim como o Delta-BNP não melhorou a previsão de risco no 120 mês de follow-up.

Conclusões: A elevação dos níveis plasmáticos de BNP nas primeiras 24h após IAM, assim como o aumento nos níveis de BNP nos dias subsequentes ao evento isquêmico, estão associados a piores desfechos no curto prazo em pacientes Killip I. Entretanto, apenas o Delta-BNP melhora a reclassificação de risco no curto prazo, tanto quando comparado ao escore GRACE como comparado ao GRACE + BNP no D1. 
ABSTRACT

Background: Available clinical scores for risk prediction after acute myocardial infarction (AMI) have a limited potential to discriminate high-risk patients. Although BNP levels at admission can predict the chance of adverse outcomes after AMI, there is no information regarding the changes in BNP levels in risk prediction of patients with AMI and normal ventricular function at admission (Killip I).

Objective: Evaluate BNP and GRACE in patients with STEMI in a prospective cohort, specifically in those with Killip I at hospital presentation.

Methods: Consecutive patients with ST-elevation AMI and Killip I ( $n=167)$ were followed for 12 months. We evaluated plasma levels of BNP in the first $24 \mathrm{~h}$ after symptoms onset (D1) and at the $5^{\text {th }}$ day (D5) and calculated the GRACE score based on admission data.

Results: Independent risk factors were selected using bootstrapping based on Coxregression analysis. Sex, age, dose of statin and diagnosis of diabetes were selected as significant predictors of sudden death and AMI for the final multivariable model. Both in crude and adjusted Cox-regressions, a Delta-BNP above the median (80pg/dL) and a BNP at D1 above 100pg/dL (median) were associated with higher incidence of sudden death and $\mathrm{AMl}$ at 30 days (OR:10.88, 95\%Cl: 1.10-108, p=0.038; OR:1.91, 95\%Cl: 1.01-4.11, p=0.049, respectively). GRACE score alone showed a moderate Cstatistic $=0.709(p=0.029)$, but adding Delta-BNP improved risk discrimination ( $C$ statistic $=0.831, p=0.001)$, with significant differences between AUCs $(p=0.046)$. Net reclassification (category-free $\mathrm{NRI}$ ) confirmed a significant improvement in individual risk prediction by $33.4 \%(95 \% \mathrm{Cl}$ : 8-61\%) compared with GRACE alone ( $\mathrm{p}=0.034)$, with adequate calibration according Bayes information criterion and Likelihood ratio test. Delta-BNP+GRACE improved reclassification especially in intermediate risk patients [by $62.5 \%(p=0.0231)$ ]. However, considering only the first measurement, BNP at D1 did not improve risk reclassification at 30 days compared to GRACE score (cfNRI $\mathrm{p}=0.8$ ), nor Delta-BNP has improved risk prediction at 12-month follow-up.

Conclusions: Both high BNP levels in the first $24 \mathrm{~h}$ after AMl and increasing levels of BNP levels in the days following were associated with poorer short-term outcomes in Killip I patients. However, only Delta-BNP improves risk reclassification in the shortterm, compared to GRACE score as well compared to GRACE + BNP at D1. 


\section{LISTA DE FIGURAS}

Figura 1: Incidência de Infarto do Miocárdio ajustada por idade e sexo, de 1999 a 2008 nos Estados Unidos. Barras "I" representam o intervalo de confiança de 95\%. "IM" indica Infarto do Miocárdio, IAMCSST indica Infarto do Miocárdio com Supradesnivelamento de Segmento ST

Figura 2: Calculadora do escore GRACE extraída do website do estudo

(http://www.outcomes-umassmed.org/grace/acs risk/acs risk content.html) 19

Figura 3: Histograma da frequência relativa da diferença entre o BNP à admissão e no quinto dia pós-infarto (Delta-BNP).

Figura 4: Modelo de Kaplan-Meier demonstrando a diferença de sobrevida livre de eventos entre os grupos com Delta-BNP abaixo e acima da mediana $(p=0.039) \ldots . .33$ Figura 5: Spline de regressão cúbica ajustada para sexo, idade, Diabetes Mellitus e logBNPD1, demonstrando a correlação existente entre o Delta BNP e a incidência de eventos (Morte Súbita cardíaca e IAM fatal/não-fatal) em 30 dias ( $p$ for trend= $0.01)$

Figura 6: Spline de regressão cúbica ajustada para sexo, idade, Diabetes Mellitus e logBNPD1, demonstrando a correlação existente entre o Delta BNP e a incidência de eventos (Morte Súbita cardíaca e IAM fatal/não-fatal) em 12 meses ( $p$ for trend=

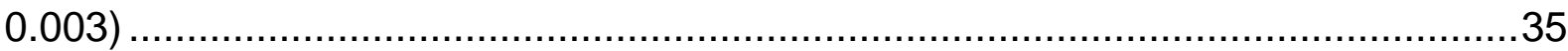

Figura 7: Regressão Linear // Delta-BNP x BNP D1 - R=0.035 ( $p=0.327) \ldots \ldots \ldots \ldots . . . .36$

Figura 8: Regressão linear // BNP D5 x BNP D1 - R=0.487 $(p<0.001) \ldots \ldots \ldots \ldots \ldots \ldots . . . . . . . .36$

Figura 9: Regressão linear // Delta-BNP x BNP D5 - R=0.893 $(p<0.001) \ldots \ldots \ldots \ldots . . . . .37$

Figura 10: Curva ROC para discriminação de Morte Súbita e IAM fatal/não fatal em 30 dias. A área sob a curva (AUC) para o modelo combinado do escore GRACE + Delta-BNP [0.823 (IC 95\% 0.67-0.98; $p=0.001)$ ] foi significativamente maior quando comparada às AUCs para o escore GRACE isolado [0.717 (95\% Cl 0.53-0.90; $\mathrm{p}=0.029)$ ] ou para o Delta-BNP [0.731 (95\% Cl 0.56-0.90; $\mathrm{p}=0.020)$ ]. TPR: True positive rate (Taxa de Verdadeiros Positivos); FPR: false positive rate (Taxa de Falsos Positivos). 


\section{LISTA DE TABELAS}

Tabela 1 - Características clínicas e laboratoriais dos pacientes, de acordo com o Delta-BNP (valores acima ou abaixo da mediana) ............................................28

Tabela 2 - Incidência de Eventos ...................................................................

Tabela 3 - Regressão Logística Binária considerando a incidência combinada de Morte Súbita e IAM fatal/não-fatal (MACE) em 30 dias como variável dependente (n $=145)$

Tabela 4 - Modelos de regressão de Cox e de Kaplan-Meier com incidência de MACE (morte súbita cardíaca + IAM fatal/não-fatal) como desfecho em 1 ano ( $\mathrm{n}=$ 145) 32

Tabela 5 - Comparação dos modelos utilizando o escore GRACE isoladamente versus o escore GRACE associado ao Delta-BNP (GRACE + Delta-BNP) para discriminação de Morte Súbita Cardíaca e IAM fatal/não-fatal. 39

Tabela 6 - Tabela de Melhora de Reclassificação 


\section{LISTA DE ABREVIATURAS E SIGLAS}

ACC - American College of Cardiology / Colégio Americano de Cardiologia AHA - American Heart Association / Associação Americana do Coração Al - Angina Instável

ANCOVA - Análise de Covariância

AUC - Area Under Curve / Área sob a curva

AVE - Acidente Vascular Encefálico

BHS - Brasília Heart Study

BIC - Bayes Information Criterion / Critério de Informação Bayesiano

BNP - Brain Natriuretic Peptide / Peptídeo Natriurético Cerebral

BNP-D1 - Dosagem de BNP à admissão hospitalar ou primeiro dia pós-infarto

BNP-D5 - Dosagem de BNP no quinto dia pós-infarto

BRA - Bloqueador do Receptor de Angiotensina

cfNRI - category-free Net Reclassification Improvement / Melhora da Reclassificação

Líquida livre de categorias

CID 10 - Classificação Internacional de Doenças, 10ª Revisão

CK-MB - Creatina Quinase, fração MB

cTnl - Cardiac Troponin I / Troponina Cardíaca I

Curva ROC - Receiver Operating Characteristic curve / Curva de Característica de Operação do Receptor

D1 - Admissão hospitalar / primeiro dia pós-infarto

D5 - quinto dia pós-infarto

D30 - trigésimo dia pós-infarto

DAC - Doença Arterial Coronariana

Delta-BNP - Variação das dosagens de BNP entre o primeiro e o quinto dias pósinfarto

DM - Diabetes Mellitus

DP - Desvio padrão.

FE - Fração de Ejeção

FNT- $\alpha$ - Fator de Necrose Tumoral $\alpha$

FPR - False positive rate / Taxa de Falsos Positivos 
GRACE - Global Registry of Acute Coronary Events / Registro Global de Eventos Coronarianos Agudos / Estudo multicêntrico de síndromes coronarianas agudas

GRE - Gradiente de Eco

HbA1c - Hemoglobina Glicada

HDL-C - High Density Lipoprotein Cholesterol / Colesterol de Lipoproteína de alta densidade

HR - Hazard Ratio

hs-cTn - high sensitivity cardiac Troponin / Troponina Cardíaca Ultrassensível

IAM - Infarto Agudo do Miocárdio

IAMCSST - Infarto Agudo do Miocárdio com supradesnivelamento do segmento ST

IAMSSST - Infarto Agudo do Miocárdio sem supradesnivelamento do segmento ST

IC - Intervalo de Confiança

ICC - Insuficiência cardíaca congestiva

IDI - Integrated Discrimination Improvement / Melhora Integrada de Discriminação

iECA - Inibidor da Enzima Conversora de Angiotensina

IMC - Índice de Massa Corporal

Killip - Classificação de Killip-Kimball

LDL-C - Low Density Lipoprotein Cholesterol / Colesterol Lipoproteína de Baixa Densidade

MACE - Major Adverse Cardiac Events / Eventos cardíacos adversos maiores (Desfecho composto de Morte Súbita e Infarto do Miocárdio)

n - Número de indivíduos compondo a amostra

MI - Myocardial Infarction / Infarto do Miocárdio (IAM)

MS - Morte Súbita

Non-STEMI - non ST-elevation Myocardial Infarction / Agrupamento dos eventos coronarianos agudos sem elevação de segmento ST

NRI - Net Reclassification Improvement / Melhora da Reclassificação Líquida

OR - Odds ratio / Razão de chances

p - Significância Estatística

PAD - Pressão arterial diastólica

PAS - Pressão arterial sistólica

PCR - Proteína C Reativa

RM - Ressonância Magnética

SCA - Síndrome Coronariana Aguda 
SBC - Sociedade Brasileira de Cardiologia

STEMI - ST-elevation Myocardial Infarction / Infarto do Miocárdio com supradesnivelamento do segmento ST (IAMCSST)

TFG - Taxa de filtração glomerular

TPR - True positive rate / Taxa de Verdadeiros Positivos

VE - Ventrículo esquerdo 


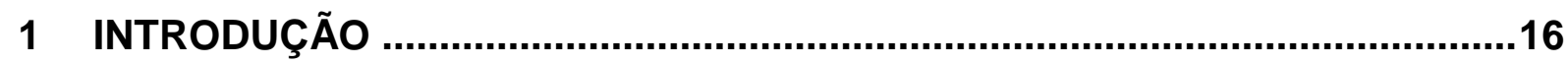

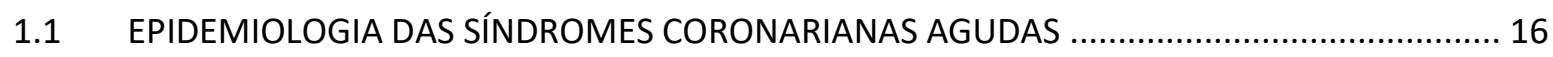

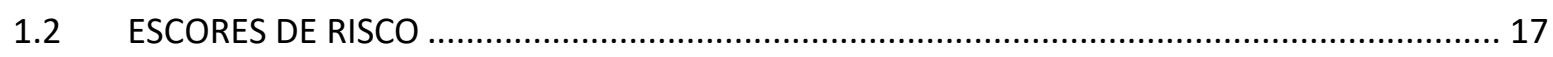

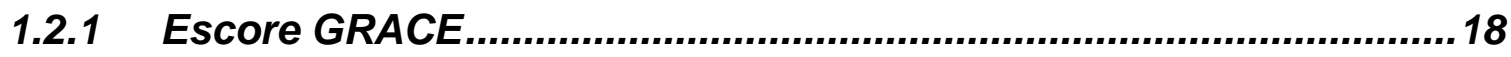

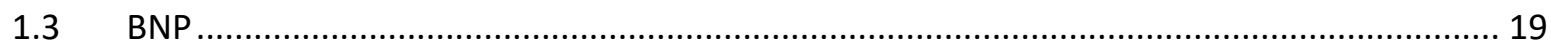

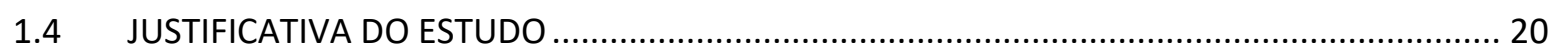

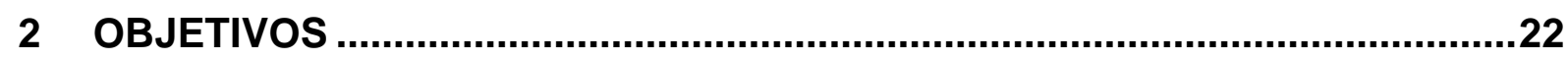

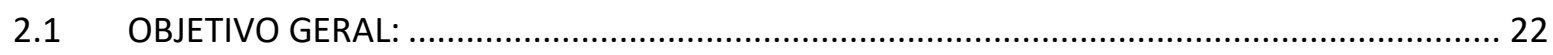

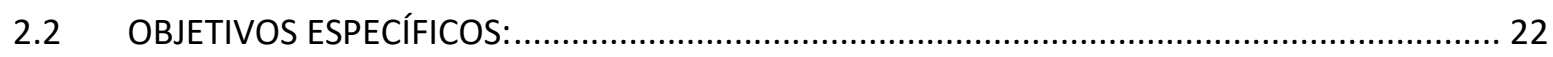

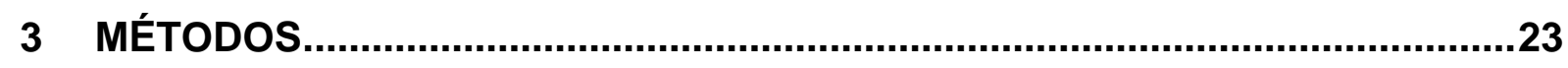

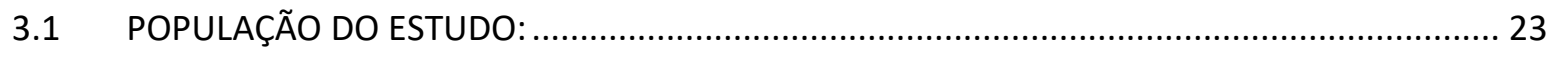

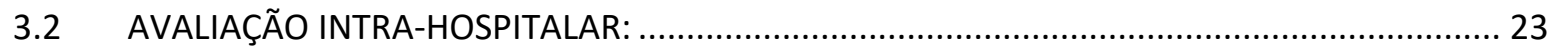

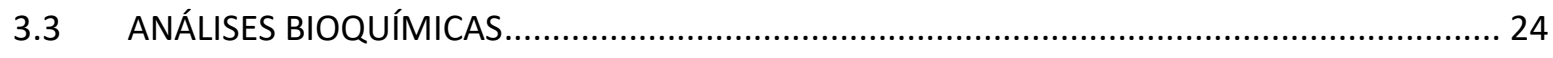

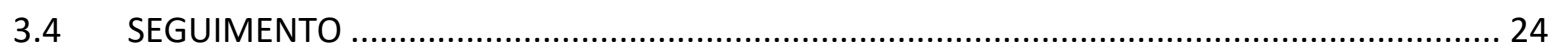

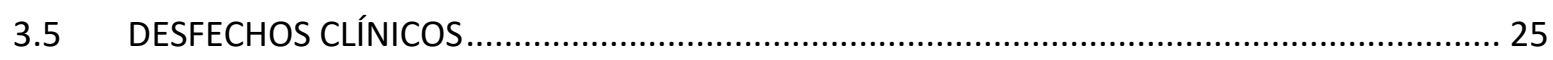

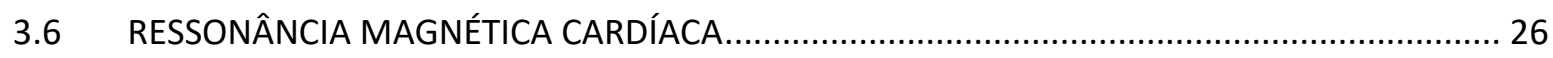

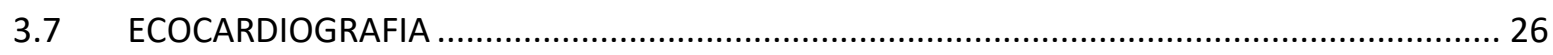

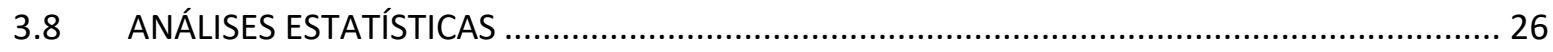

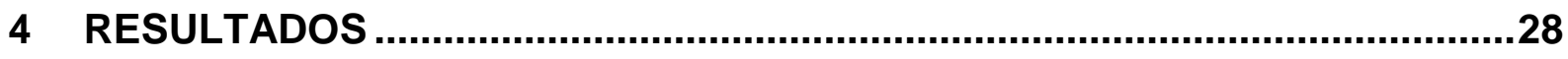

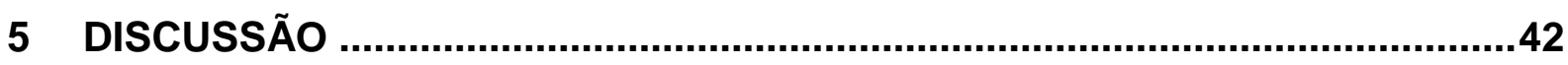

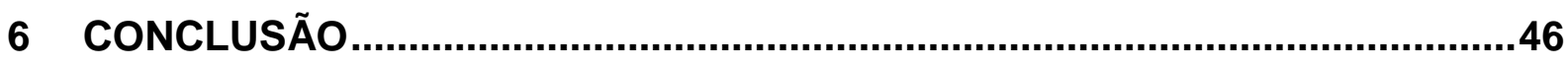

REFERÊNCIAS...................................................................................... 47

7 ANEXO A - Parecer Favorável do Comitê de Ética em Pesquisa da

Secretaria de Saúde do Distrito Federal ......................................................50 


\section{INTRODUÇÃo}

\subsection{EPIDEMIOLOGIA DAS SÍNDROMES CORONARIANAS AGUDAS}

As doenças do aparelho cardiovascular são consideradas a maior causa global de morte, sendo responsáveis por 17,5 milhões de óbitos no ano de 2012, o que representa cerca de $31 \%$ de todos os óbitos. Dentre essas mortes, estima-se que 7,4 milhões foram causadas por doença coronariana. (1)

No Brasil, de acordo com as estatísticas mais recentes (do ano de 2013), registrou-se 339.672 mortes por doenças do aparelho circulatório, o que representa cerca de $28,06 \%$ de todos os 1.210 .474 óbitos declarados, evidenciando-se como a maior causa de óbito no país. Considerando se apenas a entidade clínica Infarto do Miocárdio (CID 10 - I 21), observou-se 85.939 falecimentos (cerca de 7,09\% do total de óbitos). (2)

No Distrito Federal, unidade federativa na qual os pacientes deste estudo foram arrolados, ocorreram 11.408 óbitos no ano de 2013, dos quais 3.120 (27,34\%) foram atribuídos a causas cardiovasculares, sendo 509 relacionados a Infarto do Miocárdio (4,98\% do total de falecimentos por residência) (2)

A incidência de infarto do miocárdio com supradesnivelamento do segmento ST (IAMCSST) apresenta-se em queda ao longo das últimas décadas, estimando-se que represente cerca de 25 a $40 \%$ dos casos de infarto do miocárdio (IAM). A letalidade do IAMCSST também se apresenta em descenso, sendo estimada em 5 a $7 \%$ no período intra-hospitalar e de 7 a 18\% no primeiro ano após o infarto. Nos Estados Unidos da América em 2009, aproximadamente 683 mil pacientes receberam alta hospitalar com diagnóstico de Síndrome Coronariana Aguda. (3) 


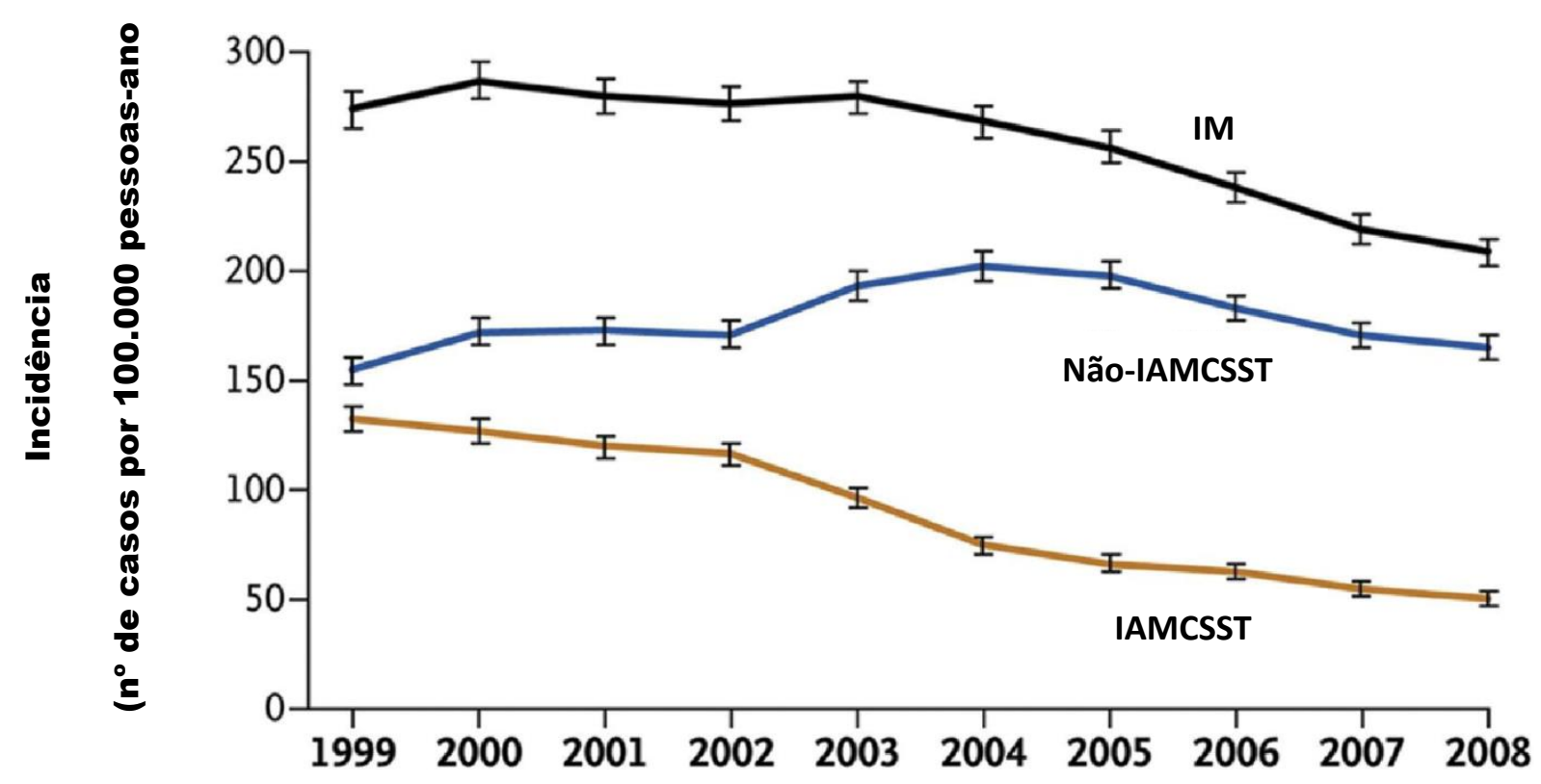

Figura 1: Incidência de Infarto do Miocárdio ajustada por idade e sexo, de 1999 a 2008 nos Estados Unidos. Barras "I" representam o intervalo de confiança de 95\%. "IM" indica Infarto do Miocárdio, IAMCSST indica Infarto do Miocárdio com Supradesnivelamento de Segmento ST

Retirado de: O'Gara PT, Kushner FG, Ascheim DD, Casey DE, Chung MK, de Lemos JA, et al. 2013 ACCF/AHA Guideline for the Management of ST-Elevation Myocardial InfarctionA Report of the American College of Cardiology Foundation/American Heart Association Task Force on Practice Guidelines. Journal of the American College of Cardiology. 2013;61(4):e78-e140

\subsection{ESCORES DE RISCO}

Ferramentas de avaliação de risco são importantes na identificação de pacientes com maior probabilidade de desenvolver eventos fatais ou potencialmente incapacitantes e que, eventualmente, possam se beneficiar de cuidados mais especializados e/ou estratégias de tratamento mais agressivos, possivelmente diminuindo a morbimortalidade das Síndromes Coronarianas Agudas (SCAs). (3) 


\subsubsection{Escore GRACE}

O GRACE - Global Registry of Acute Coronary Events (em português, Registro Global de Eventos Coronarianos Agudos) foi um estudo prospectivo multinacional que ocorreu de 1999 a 2009, do qual participaram 247 hospitais em 30 países, dentre eles o Brasil (7 centros), com um total de 102.341 pacientes arrolados.(4) O critério de inclusão do estudo consistia de pacientes adultos (idade maior que 18 anos) com história clínica compatível para SCA e pelo menos uma das seguintes alterações: mudanças eletrocardiográficas compatíveis com SCA, aumentos seriados de marcadores de necrose miocárdica, doença arterial coronariana documentada. Pacientes cuja sintomatologia clínica à admissão fosse atribuída a causa não cardiovascular eram excluídos, bem como o eram pacientes cujo diagnóstico final não fosse de SCA.(5)

A partir do estudo supracitado, desenvolveu-se um modelo de escore de risco baseado em parâmetros clínicos simples e biomarcadores facilmente disponíveis.(6) Dispondo-se de dados admissionais como idade, frequência cardíaca, pressão arterial sistólica, creatinina sérica, classificação de Killip-Kimball, alteração de segmento ST, parada cardíaca e elevação de marcadores de necrose miocárdica, e utilizando uma calculadora especial de livre acesso disponibilizada pelo estudo em seu website ou na forma de aplicativos para celular, é possível estimar o risco de morte e novo infarto do miocárdio durante o período intra-hospitalar e até 6 meses após o evento coronariano. (Figura 2)

O escore de risco GRACE é validado para uso nas síndromes coronarianas agudas em suas diversas apresentações (IAMCSST, IAMSSST, AI), bem como em diferentes populações, sendo reconhecido como um robusto preditor de morte e outros eventos adversos em pacientes com SCA. (7-10) Especificamente em relação aos pacientes com IAMCSST, o escore GRACE de mortalidade intra-hospitalar demonstra uma boa capacidade de predição de óbito em 30 dias pós-infarto. Apesar disso, nota-se que o mesmo tende a ser mais preciso em pacientes com risco mais alto, tais como os classificados como Killip II a IV, comparados a pacientes sem sinais clínicos de insuficiência cardíaca à admissão hospitalar pós-infarto (Killip I) (11, 12). 


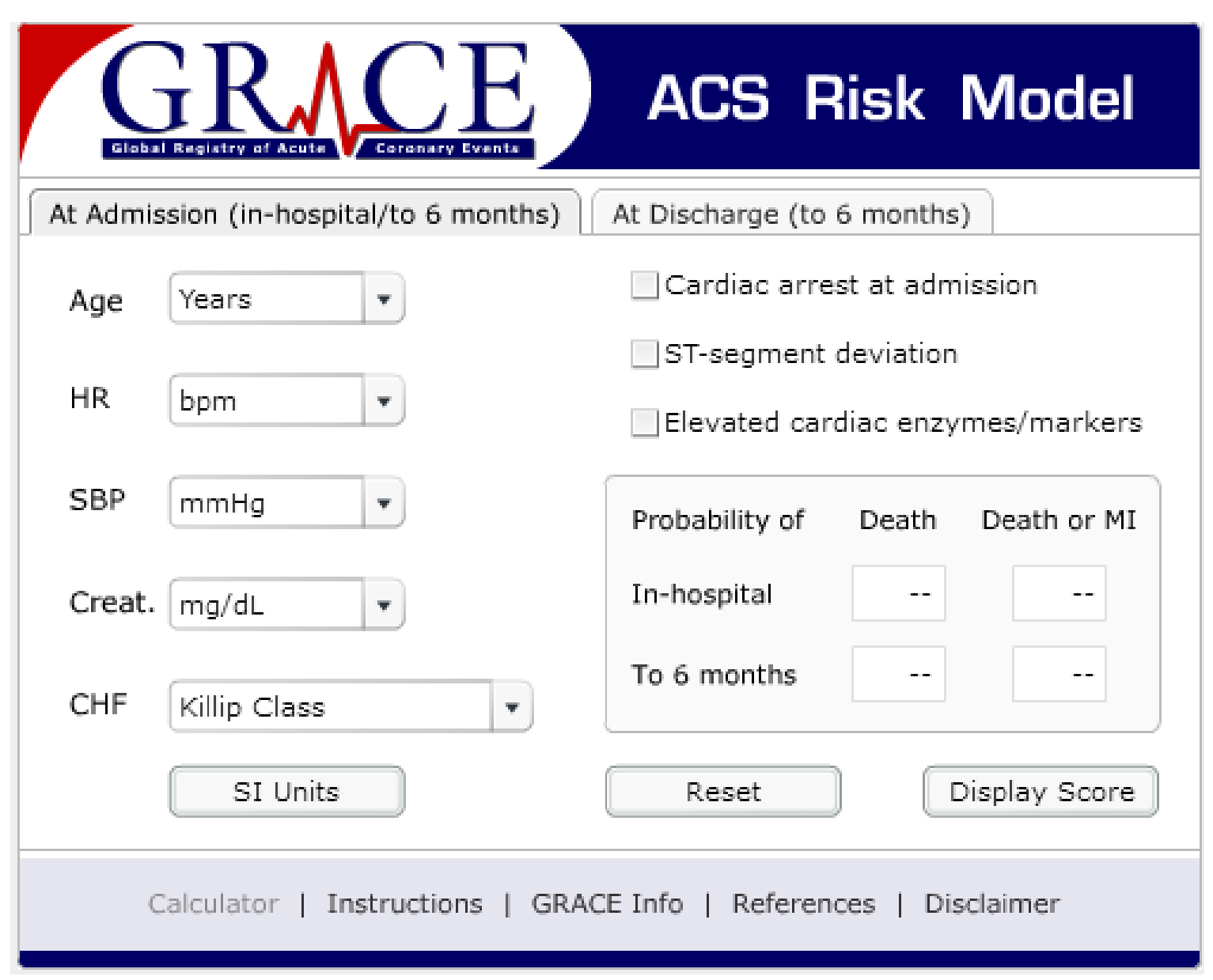

Figura 2: Calculadora do escore GRACE extraída do website do estudo (http://www.outcomes-umassmed.org/grace/acs risk/acs risk content.html)

\subsection{BNP}

O peptídeo natriurético do tipo B ou cerebral (BNP - Brain Natriuretic Peptide) é um polipeptídeo secretado pelos ventrículos cardíacos em resposta à dilatação ventricular e sobrecarga de pressão. (13) As concentrações séricas de BNP refletem o grau de disfunção ventricular esquerda e possuem significância prognóstica após SCA, porém também podem aumentar em pacientes com IAM e função ventricular relativamente normal ou fração de ejeção preservada. (14) Medidas de BNP nos primeiros dias pós-infarto são capazes de predizer risco de morte, insuficiência cardíaca e novo IAM. $(14,15)$ Em um estudo com 483 pacientes do ensaio clínico 
randomizado ENTIRE-TIMI-23, BNP à admissão > 80pg/ml foi independentemente associado a maior risco de morte em 30 dias após IAMCSST. (16)

Após o infarto do miocárdio, as concentrações séricas de BNP se elevam rapidamente durante o primeiro dia, apresentando pico cerca de 20h após o início dos sintomas, e depois tendendo a se estabilizar. $(13,17)$ Entretanto, são descritos dois padrões de elevação deste marcador: um padrão monofásico, com uma redução da concentração sérica de BNP nas 48 a 72h subsequentes ao pico inicial, e um padrão bifásico, ocorrendo em cerca de metade dos pacientes, caracterizado por um segundo pico de BNP por volta do quinto dia pós-IAM.(17) Este último padrão, bifásico, parece refletir o processo de remodelamento ventricular, e está associado a maior risco de morte, insuficiência cardíaca e novo infarto do miocárdio.(14, 15, 17)

\subsection{JUSTIFICATIVA DO ESTUDO}

Estudos anteriores buscaram avaliar se a adição do BNP ao escore GRACE resultaria em melhora do poder de predição de eventos adversos nas síndromes coronarianas agudas, porém os resultados foram conflitantes. Alguns trabalhos encontraram uma melhora na estimativa de risco em pacientes com síndromes coronarianas sem elevação de segmento ST(18) e quando o espectro das síndromes coronarianas agudas foi avaliado conjuntamente $(19,20)$, ao passo que outro estudo não evidenciou qualquer benefício adicional da combinação de BNP ou troponina cardíaca ultrassensível (hs-cTn) ao escore GRACE em termos de refinamento de predição de risco (21). Um dos pontos a ser considerado é que, apesar de bem conhecidos os padrões monofásico e bifásico de ascensão e queda das concentrações séricas de BNP no pós-infarto, nenhum dos trabalhos avaliando a adição do BNP a escores clínicos se atentou a estas particularidades, o que pode ter motivado a inconsistência observada nos resultados, possivelmente subestimando o poder preditivo do BNP no infarto do miocárdio.

Apesar dos estudos descritos, ainda existem muito poucas análises abordando o valor da adição do BNP ao escore GRACE na predição de mortalidade e eventos cardíacos adversos (MACE - Major Adverse Cardiac Events) em pacientes com IAMCSST, a entidade de maior risco dentro das síndromes coronarianas agudas.(20) 
Não obstante, é notável a relativa ausência de estudos no subgrupo de pacientes com IAMCSST sem disfunção ventricular associada à admissão hospitalar (Killip I). Faz-se necessário distinguir em um grupo considerado, a priori, como de baixo risco (Killip I) - risco de morte de 6\%(22)) aqueles indivíduos com maior probabilidade de evoluir desfavoravelmente, para que estes possam porventura se beneficiar de alguma medida que vise a redução de seu risco de apresentar quaisquer desfechos adversos. Portanto, o aperfeiçoamento das ferramentas de estimativa de risco nestes pacientes ainda é uma necessidade clínica atual a ser atingida e que deve ser melhor explorada. 


\section{OBJETIVOS}

\subsection{OBJETIVO GERAL:}

Avaliar capacidade do escore GRACE associado a medidas de BNP para predição de eventos cardiovasculares adversos em pacientes com infarto do miocárdio com supradesnivelamento do segmento ST sem disfunção ventricular clinicamente evidente à admissão hospitalar (Killip I)

\subsection{OBJETIVOS ESPECÍFICOS:}

- Avaliar e comparar performance entre BNP isolado, GRACE isolado, associação GRACE + BNP na predição de eventos cardiovasculares adversos pós infarto

- Avaliar associação entre variação de BNP (Delta-BNP) e incidência de eventos cardiovasculares (sobrevida livre de eventos) 


\section{MÉTODOS}

\subsection{POPULAÇÃO DO ESTUDO:}

A amostra consiste de 167 pacientes prospectivamente admitidos no estudo Coorte Brasília / Brasília Heart Study (BHS) (ClinicalTrials.gov Identifier: NCT02062554) de Maio de 2006 a Abril de 2010. O BHS, ainda em andamento, é uma coorte prospectiva observacional de pacientes com IAMCSST admitidos no Hospital de Base do Distrito Federal (centro de referência para síndromes coronarianas agudas de Brasília, DF). Os critérios de elegibilidade para inclusão no estudo são os seguintes: (I) menos de $12 \mathrm{~h}$ após o início dos sintomas de infarto, (II) elevação do segmento ST de pelo menos $1 \mathrm{~mm}$ no plano frontal ou $2 \mathrm{~mm}$ no plano horizontal em duas derivações contíguas do eletrocardiograma de 12 derivações, (III) necrose miocárdica evidenciada por elevação de pelo menos um valor acima do percentil 99 do limite de referência de CK-MB (i.e. 25 U/L) e Troponina I (i.e. 0,04 ng/mL) seguidos por declínio de ambos, e (IV) ausência de impedimentos para o seguimento clínico. $\mathrm{O}$ estudo foi aprovado pelo Comitê de Ética da Fundação de Ensino e Pesquisa em Ciências da Saúde (FEPECS) da Secretaria de Saúde do Distrito Federal sob protocolo de número 130/2006, e todos os pacientes incluídos assinaram o Termo de Consentimento Livre e Esclarecido, autorizando sua participação no estudo.

\subsection{AVALIAÇÃO INTRA-HOSPITALAR:}

Uma avaliação médica geral e completa foi realizada em todos os pacientes à admissão hospitalar (D1). Amostras de sangue eram coletadas de cada paciente no D1 e após 5 dias do IM (D5) e centrifugadas por 10min a 3500rpm. Alíquotas de plasma eram retiradas e congeladas a $-80{ }^{\circ} \mathrm{C}$ para conservação do material e posteriores análises. As condutas médicas terapêuticas durante o período intrahospitalar eram decididas pelos médicos assistentes do Hospital de Base do Distrito 
Federal, sem interferência dos pesquisadores, e seguiam um protocolo baseado nas diretrizes da Sociedade Brasileira de Cardiologia (SBC), American Heart Association (AHA)/American College of Cardiology (ACC) para IAMCSST.

\subsection{ANÁLISES BIOQUÍMICAS}

As seguintes análises foram realizadas: glicose (Glucose GOD-PAP, Roche Diagnostics, Mannheim, Germany), colesterol total (Glucose GOD-PAP, Roche Diagnostics, Mannheim, Germany), triglicerídeos (GPO-PAP, Roche Diagnostics, Mannheim, Germany), colesterol HDL (High-Density Lipoprotein Cholesterol, HDL-c) (Roche Diagnostics, Mannheim, Germany), hemoglobina glicosilada (HbA1c) (Variant II, Bio-Rad Laboratories, Hercules, CA, USA), Troponina Cardíaca I (cTnl) (ADVIA Centaur XP immunoanalyzer, Siemens Healthcare Diagnostics). O colesterol LDL (Low Density Lipoprotein Cholesterol, LDL-C) foi calculado utilizando a fórmula de Friedewald.

A concentração plasmática de BNP foi medida por método ELISA disponível comercialmente (BNP-32 Human EIA kit, Phoenix Pharmaceuticals CA, USA). Com o objetivo de avaliar a elevação subsequente do BNP como variável contínua e para balancear sua interação com os desfechos, foi calculada a variação de BNP (DeltaBNP) subtraindo-se o valor do BNP no D1 do valor no D5. A variação dos valores de outros parâmetros bioquímicos também foi calculada como a diferença relativa entre o D5 e D1 (Delta).

\subsection{SEGUIMENTO}

Antes da alta hospitalar, todos os pacientes foram orientados quanto à adequação da dieta, cessação do hábito tabágico, manutenção de atividades físicas regulares e perda ponderal, e receberam prescrição médica de acordo com as recomendadas pelas diretrizes. Após isso, os pacientes foram acompanhados 
ambulatorialmente no BHS, com consultas a cada 15-45 dias. As prescrições de seguimento incluíam: estatinas, com adição de ezetimibe quando necessário (meta de colesterol LDL de $70 \mathrm{mg} / \mathrm{dL}$ ); inibidores da enzima conversora de angiotensina (iECA) ou bloqueadores do receptor de angiotensina (BRA) para hipertensão ou se redução da fração de ejeção do ventrículo esquerdo (<40\%); controle glicêmico (HbA1c < 7,0\%); clopidogrel 75mg/dia; ácido acetilsalicílico 100mg/dia; terapia antiisquêmica conforme necessidade (propranolol e dinitrato de isossorbida, sozinhos ou em combinação). Os médicos envolvidos no seguimento clínico foram encobertos em relação a todas as análises realizadas no estudo.

\subsection{DESFECHOS CLÍNICOS}

A avaliação dos desfechos clínicos era realizada ambulatorialmente a cada 30 dias. Pacientes impossibilitados de comparecer às consultas ou parentes de pacientes falecidos eram contatados por telefone. Informações sobre as causas de morte e eventos clínicos eram obtidas da declaração de óbito ou registros de prontuários médicos. Os seguintes eventos adversos cardíacos foram considerados: morte súbita cardíaca e recorrência de IAM. Morte súbita cardíaca foi definida como evento inesperado e não traumático ocorrendo no intervalo de $6 \mathrm{~h}$ do início dos sintomas. Infarto do Miocárdio fatal se refere a qualquer morte precedida por infarto em fase aguda. Novo IAM foi definido como internação hospitalar documentada com sintomas consistentes com infarto agudo do miocárdio e elevação de CK-MB e Troponina I (um valor acima do percentil 99 do valor de referência) com padrão compatível com IAM. Quando o paciente apresentou mais de um evento, a primeira ocorrência foi selecionada. Os dados de pacientes que foram a óbito por causas não cardíacas foram censurados no dia de sua morte. 


\subsection{RESSONÂNCIA MAGNÉTICA CARDÍACA}

A Ressonância Magnética (RM) cardíaca foi realizada em aparelho de 1.5T GE CV/i (Wakeusha, Wisconsin) 15 dias após o IAM. Um gradiente eco (GRE) de inversão-recuperação foi usado para estimativa da massa infartada por realce tardio (10 a 20min após injeção intravenosa de $0.2 \mathrm{mmol} / \mathrm{kg}$ de contraste a base de gadolínio). As imagens foram avaliadas por um radiologista experiente que não tinha acesso a qualquer dado do estudo (encoberto).

\subsection{ECOCARDIOGRAFIA}

Avaliação ecocardiográfica foi realizada no quinto dia pós-IM (D5) e no trigésimo dia após o evento (D30). A fração de ejeção (FE) ventricular esquerda foi estimada utilizando o método de Simpson a partir de imagem biplanar apical de duas e quatro câmaras, de acordo com as recomendações da American Society of Echocardiography e da European Association of Cardiovascular Imaging (23). O exame foi realizado utilizando-se imagens bidimensionais e efeito Doppler (HDI 5000, Philips ATL, Bothell, WA).

\subsection{ANÁLISES ESTATÍSTICAS}

Os dados com distribuição normal são apresentados como média \pm desvio padrão (DP) e dados com distribuição não-normal são mostrados como mediana (intervalo interquartil). Análises de covariância (ANCOVA) para o Delta-BNP foram ajustadas por sexo, idade e níveis basais. Transformação logarítmica foi utilizada às variáveis com distribuição não-normal e o teste de Kolmogorov-Smirnov usado para avaliar normalidade da distribuição. Curvas ROC (Receiver Operating Characteristic) foram construídas para avaliar o poder discriminativo do escore GRACE, do BNP e da 
combinação GRACE + BNP em relação aos desfechos estudados (morte súbita, IAM fatal e não-fatal) em 30 dias após o evento índice, e suas respectivas áreas sob as curvas (AUCs) foram comparadas utilizando o método de "bootstrapping". Também foi calculada a estatística $C$ de Harrell, possibilitando censura de dados e permitindo a avaliação da precisão preditiva do modelo com e sem BNP. Fatores de risco independentes foram selecionados utilizando "bootstrapping" baseado em análises de regressão de Cox. Sexo, idade, dose de estatinas e diagnóstico de diabetes foram selecionados como preditores significativos de morte súbita e IAM para o modelo multivariável final. Modelos ajustados de regressão logística e de Cox, bem como um modelo de "splines" cúbicas, foram utilizados para avaliar a incidência de IAM fatal/não fatal e morte súbita respectivamente no longo (12 meses) e curto (30 dias) prazo. As análises de curva ROC também foram utilizadas para descrever o poder preditivo das variáveis e para identificação de pontos de corte ótimos. Análises de reclassificação (NRI - Net Reclassification Improvement; cfNRI - category-free NRI; IDI - Integrated Discrimination Improvement) foram utilizadas para caracterização da melhora do escore GRACE padrão obtida com a adição de novos marcadores. Um valor de $p$ bicaudal $<0.05$ foi considerado estatisticamente significativo. Utilizou-se os softwares SPSS (versão 21.0 para Mac) e STATA (versão 12.0 para Mac) para toda as análises realizadas. 


\section{RESULTADOS}

As características clínicas iniciais e dados laboratoriais da admissão hospitalar dos pacientes constam na Tabela 1. A Figura 3 revela um histograma da mudança relativa do BNP plasmático do D1 para o D5.

Tabela 1 - Características clínicas e laboratoriais dos pacientes, de acordo com o DeltaBNP (valores acima ou abaixo da mediana)

\section{Delta - BNP}

$\mathrm{n}$

Idade, em anos

Sexo Masculino, \%

$\mathrm{IMC}, \mathrm{Kg} / \mathrm{m}^{2}$

Circunferência Abdominal, cm

História de DM ou Hb1Ac > 7, \%

$\mathrm{HbA1c} \%$

História de Hipertensão, \%

IAM prévio ou AVE, \%

História Familiar de DAC, \%

Dislipidemia, \%

Sedentarismo, \%

Tabagismo, \%

PAS, $\mathrm{mmHg}$

PAD, $\mathrm{mmHg}$

TFG (MDRD), $\mathrm{ml}^{*} \mathrm{~kg}^{-1} * \mathrm{~h}^{-1}$

Killip-Kimbal classe I, \%
Abaixo da

Mediana

84

$62 \pm 10$

73

$26.7 \pm 4$

$95.1 \pm 11$

36

$6.55 \pm 1.7$

60

17

43

35

56

38

$135 \pm 27$

$85 \pm 17$

$69.5 \pm 21$

100
Acima da

Mediana

83

$63 \pm 11$

0.4

73

1.0

$26.2 \pm 4$

0.6

$95.6 \pm 10$

1.0

22

0.052

$6.38 \pm 1.6$

0.6

62

0.8

15

0.7

42

0.9

37

0.8

54

0.8

42

0.6

$139 \pm 33$

0.5

$87 \pm 19$

0.4

$73.3 \pm 28$

0.4

100 


\begin{tabular}{|c|c|c|c|}
\hline Trombólise Química, \% & 74 & 79 & 0.5 \\
\hline Angioplastia Primária, \% & 13 & 10 & 0.6 \\
\hline $\begin{array}{l}\text { Tempo até terapia de reperfusão, } \\
\min \end{array}$ & $175 \pm 30$ & $190 \pm 32$ & 0.2 \\
\hline Uso de Betabloqueadores, \% & 56 & 60 & 0.7 \\
\hline Uso de Sinvastatina, \% & 72 & 70 & 0.8 \\
\hline Dose de Sinvastatina, mg/dia & $31 \pm 24$ & $25 \pm 21$ & 0.11 \\
\hline LDL-C, mg/dL & $132 \pm 42$ & $120 \pm 43$ & 0.11 \\
\hline $\mathrm{HDL}-\mathrm{C}, \mathrm{mg} / \mathrm{dL}$ & $38.3 \pm 12$ & $37.5 \pm 11$ & 0.65 \\
\hline Triglicerídeos, mg/dL & $185 \pm 95$ & $141 \pm 74$ & 0.017 \\
\hline Glicose, mg/dL & $141 \pm 74$ & $129 \pm 41$ & 0.22 \\
\hline Pico de CKMB, U/L & $202(244)$ & $177(252)$ & 0.5 \\
\hline cTnl, ng/mL & $1.57(19.2)$ & $2.80(22.0)$ & 0.7 \\
\hline Massa infartada - RM cardíaca, g & $16.5 \pm 9$ & $18.4 \pm 10$ & 0.5 \\
\hline \multicolumn{4}{|l|}{ Fração de Ejeção (Simpson), \% } \\
\hline D5 & $52.1(13)$ & $52.0(11)$ & 0.7 \\
\hline D30 & $59.0(13)$ & $52.5(14)$ & 0.040 \\
\hline Escore GRACE & $135 \pm 23$ & $138 \pm 29$ & 0.32 \\
\hline BNP no D1, pg/dL & $145(140)$ & $180(130)$ & 0.20 \\
\hline BNP no D5, pg/dL & $120(132)$ & 410 (320) & $<0.001$ \\
\hline Delta - BNP, pg/dL & $0(88)$ & $220(201)$ & $<0.001$ \\
\hline \multicolumn{4}{|l|}{ FNT- $\alpha, n g / L$} \\
\hline $\mathrm{D} 1$ & $5.6(0.2 ; 14)$ & $1.9(0.2 ; 14)$ & 0.50 \\
\hline D5 & $15.2(7.1 ; 35)$ & $12.0(4.4 ; 26)$ & 0.63 \\
\hline Delta & $7.2(1.5 ; 18)$ & $6.3(2.0 ; 14)$ & 0.95 \\
\hline \multicolumn{4}{|l|}{$\mathrm{PCR}, \mathrm{mg} / \mathrm{dL}$} \\
\hline D1 & $0.46(0.2 ; 1.2)$ & $0.60(0.3 ; 1.3)$ & 0.69 \\
\hline D5 & $2.76(1.3 ; 5.2)$ & $3.44(1.6 ; 6.2)$ & 0.59 \\
\hline Delta & $1.65(0.4 ; 4.7)$ & $2.12(0.8 ; 5.3)$ & 0.72 \\
\hline
\end{tabular}




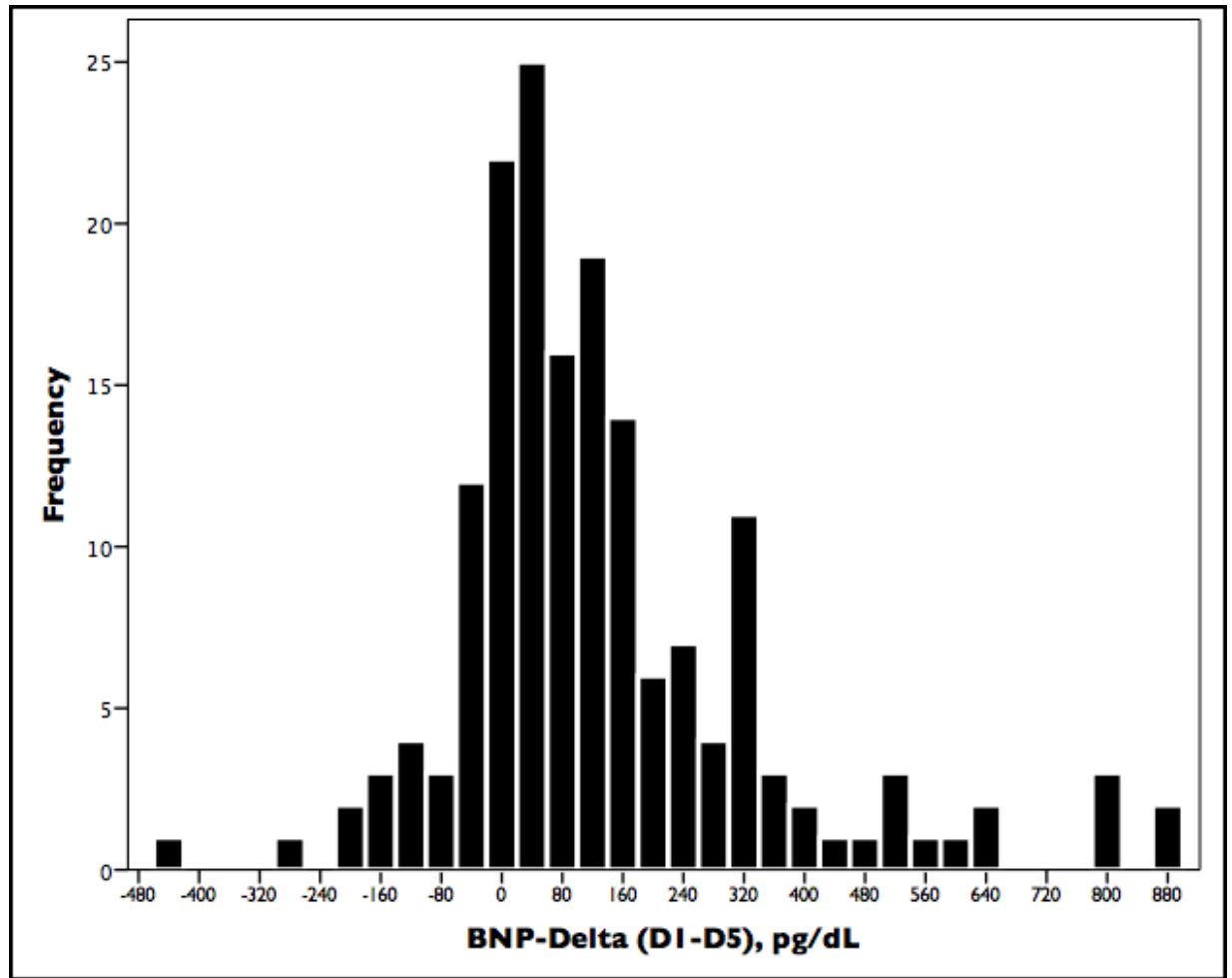

Figura 3: Histograma da frequência relativa da diferença entre o BNP à admissão e no quinto dia pós-infarto (Delta-BNP)

Ocorreram 9 eventos nos primeiros 30 dias, distribuídos em 6 infartos do miocárdio fatais e 3 mortes súbitas, e 17 eventos na avaliação em 12 meses, tendo sido 4 IAM não-fatais, 9 IAM fatais e 4 episódios de MS.

Tabela 2 - Incidência de Eventos

Eventos

Morte Súbita

IAM Fatal

IAM não-fatal
30 Dias

3

6

0

9
12 Meses

4

9

4

17


Os pacientes foram divididos em 2 grupos de acordo com a mediana do DeltaBNP (80pg/dL). Não houve diferença estatisticamente significativa entre os grupos, embora história de Diabetes Mellitus (DM), maiores concentrações plasmáticas de colesterol LDL e maior dosagem de estatina tenderam a ser mais prevalentes no grupo com Delta-BNP abaixo da mediana, sendo tais fatores incluídos na análise ajustada. Apesar de o pico de CK-MB, massa infartada estimada pela RM e fração de ejeção no D5 terem sido similares entre os grupos, a fração de ejeção no D30 foi significativamente menor nos pacientes com maior Delta-BNP [59.0(49;62) vs. 52.3(45;59), $p=0.04]$. As concentrações plasmáticas de cTnl à admissão foram similares entre os dois grupos $(p=0.7)$, bem como as concentrações de FNT- $\alpha$ e PCR.

Em modelos ajustados de regressão de Cox, as concentrações de Delta-BNP acima da mediana foram associadas a maior incidência de morte súbita e de IAM fatal/não-fatal durante os primeiros 30 dias após o IAMCSST (OR 10.88, IC 95\% 1.10108, $\mathrm{p}=0.038$ ) e em 12 meses (HR 2.51, IC 95\% 1.03-6.11, $\mathrm{p}=0.043$ ) (

Tabela 33 e a no modelo totalmente ajustado

Tabela 44).

Tabela 3 - Regressão Logística Binária considerando a incidência combinada de Morte Súbita e IAM fatal/não-fatal (MACE) em 30 dias como variável dependente $(n=145)$

OR IC 95\% para OR

$\mathrm{p}$

Inferior Superior

Delta-BNP acima da mediana vs.

Incidência de MACE em 30 dias

Não-ajustado

6.94

0.81

59

0.077

Ajustado para sexo e idade

6.82

0.80

58

0.080

Totalmente ajustado*

10.88

1.10

108

0.038

* Valor de $p$ ajustado para sexo, idade, história prévia de DM (ou HbA1c admissional > $6,5 \%)$ e dose de estatina no modelo totalmente ajustado 
Tabela 4 - Modelos de regressão de Cox e de Kaplan-Meier com incidência de MACE (morte súbita cardíaca + IAM fatal/não-fatal) como desfecho em 1 ano ( $n=145)$

\begin{tabular}{ccccc}
\hline & Regressão de Cox & Kaplan-Meier \\
\hline HR & IC 95\% para HR & Inferior Superior & $p$ & Breslow Log Rank \\
& & Sig. Sig.
\end{tabular}

Delta-BNP maior que a mediana vs.

incidência de MACE em 1 ano

$\begin{array}{lllllll}\text { Não-ajustado } & 2.21 & 0.94 & 5.23 & 0.070 & 0.063 & 0.039\end{array}$

$\begin{array}{lllll}\text { Ajustado para sexo e idade } & 2.36 & 0.98 & 5.70 & 0.056\end{array}$

$\begin{array}{lllll}\text { Totalmente ajustado* }^{*} & 2.51 & 1.03 & 6.11 & 0.043\end{array}$

* Valor de $p$ ajustado para sexo, idade, história prévia de DM (ou HbA1c admissional $>6,5 \%$ ) e dose de estatina no modelo totalmente ajustado. Tempo médio de seguimento de 345 (329 - 360) e 305 (276-333) dias nos grupos com Delta-BNP abaixo e acima da mediana, respectivamente.

A sobrevida livre de eventos, evidenciada pela curva de Kaplan-Meier (Figura 4), é significativamente menor no grupo com Delta- BNP acima da mediana. ( $p$ log rank test $=0.039$ ) 


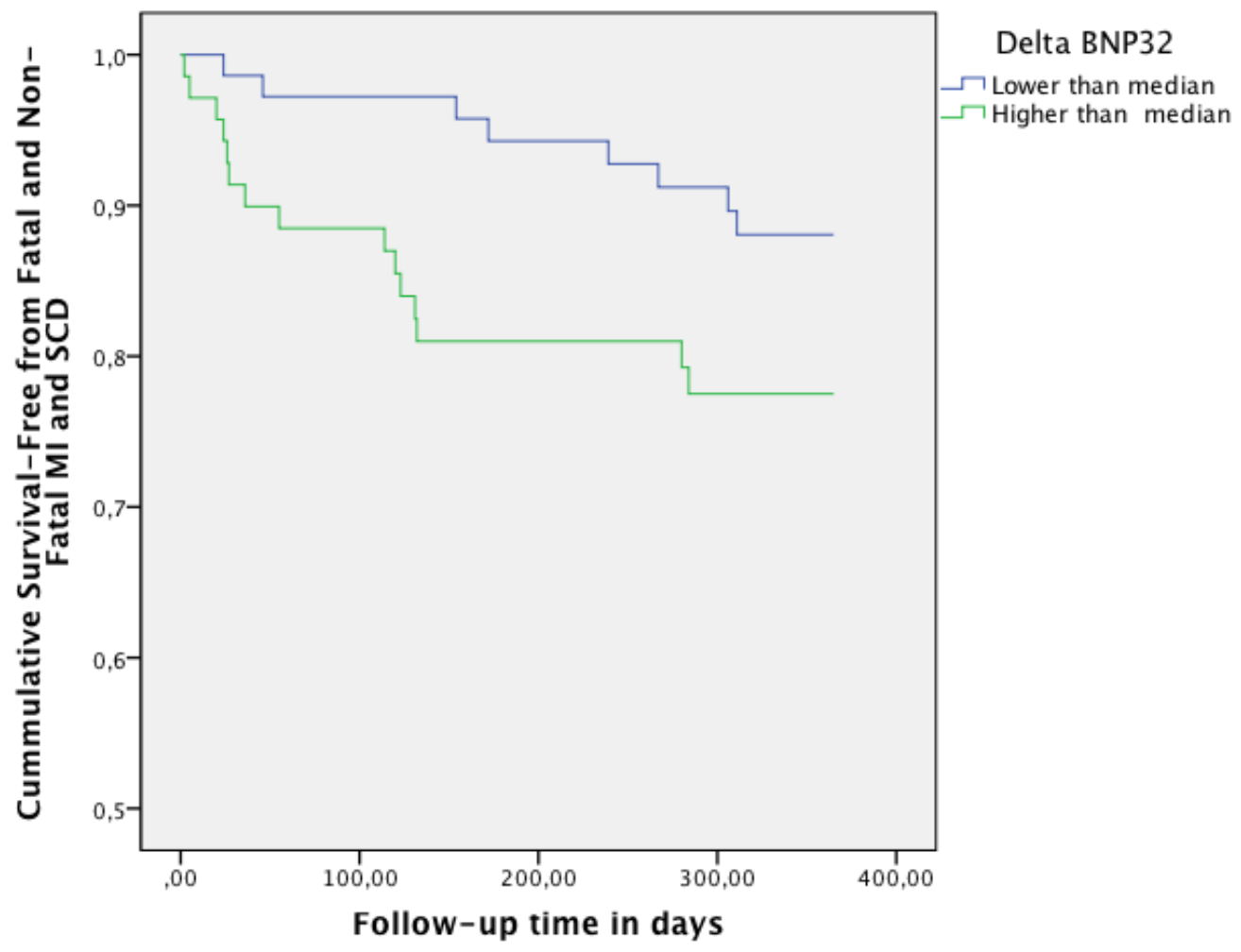

Figura 4: Modelo de Kaplan-Meier demonstrando a diferença de sobrevida livre de eventos entre os grupos com Delta-BNP abaixo e acima da mediana $(p=0.039)$

Em uma análise contínua do Delta-BNP, observa-se que este também se associa ao desfecho primário tanto em 30 dias (OR 1.032 para cada aumento de $10 \mathrm{pg} / \mathrm{dL}$, IC 95\% 1.013-1.052, $\mathrm{p}<0.001$ ) quanto em 12 meses (HR 1.021 para cada aumento de 10pg/dL, IC 95\% 1.005-1.038, $p=0.008$ ). Paralelamente, concentrações de BNP no D1 acima de 100pg/dL (mediana) foram associadas a maior incidência de MS e IAM em 30 dias (OR 1.91, IC 95\%: 1.01-4.11, $\mathrm{p}=0.049$ para análise como variável categórica, porém $\mathrm{p}=0.81$ para o BNP-D1 como variável contínua), mas não em 12 meses ( $p=0.67$ para análise como variável categórica; $p=0.60$ para BNP-D1 como variável contínua).

As concentrações admissionais de Troponina I (após transformação logarítmica) não se associaram ao desfecho primário em 30 dias $(p=0.4)$ ou em 12 meses $(p=0.6)$.

No modelo ajustado de regressão de splines cúbicas, comparou-se a incidência de eventos (em pacientes-ano) de acordo com o Delta-BNP tanto para 30 dias como para 12 meses. Como evidenciado pela Figura 5 e Figura 6, há uma correlação 
positiva e estatisticamente significativa entre a incidência de eventos e a variável preditora Delta-BNP ( $p$ para tendência em 30 dias $=0.01 ; p$ para tendência em 12 meses $=0.003$ ).

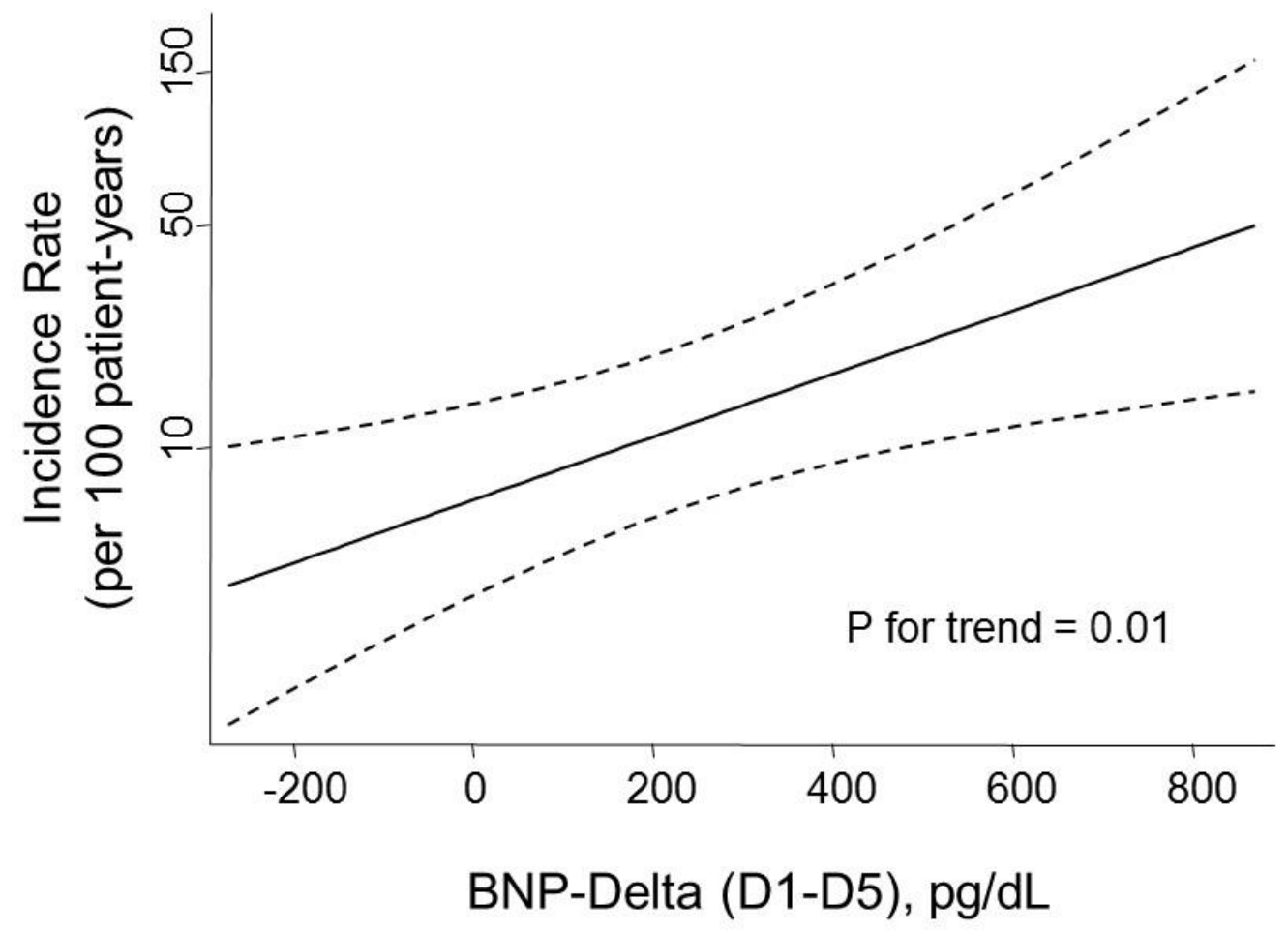

Figura 5: Spline de regressão cúbica ajustada para sexo, idade, Diabetes Mellitus e logBNPD1, demonstrando a correlação existente entre o Delta BNP e a incidência de eventos (Morte Súbita cardíaca e IAM fatal/não-fatal) em 30 dias ( $p$ for trend $=$ 0.01) 


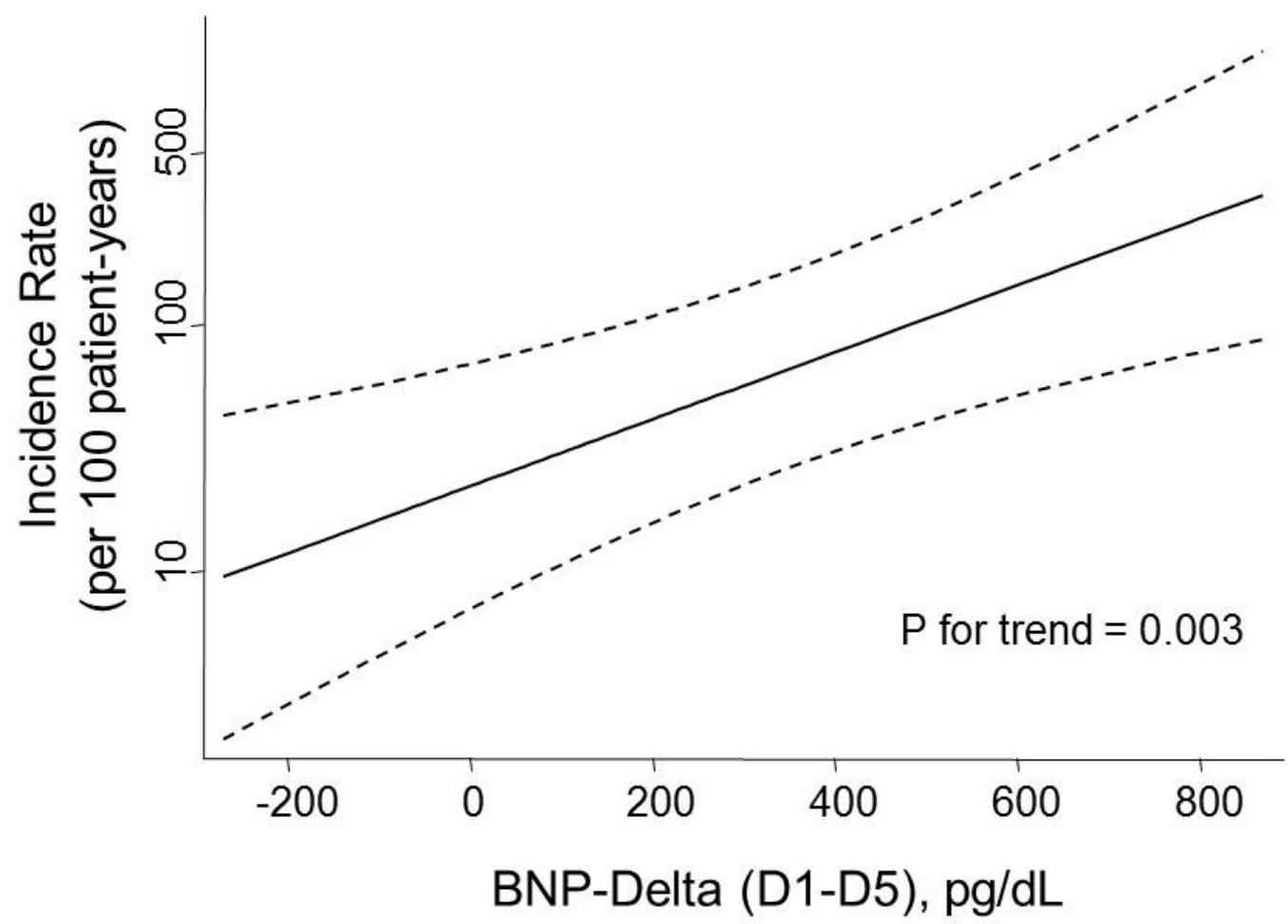

Figura 6: Spline de regressão cúbica ajustada para sexo, idade, Diabetes Mellitus e logBNPD1, demonstrando a correlação existente entre o Delta BNP e a incidência de eventos (Morte Súbita cardíaca e IAM fatal/não-fatal) em 12 meses ( $p$ for trend = 0.003)

Avaliando-se os gráficos de regressão linear, observa-se que não houve correlação entre as variáveis Delta-BNP e BNP D1 [Figura 7, coeficiente de correlação $R=0.035$ ( $p=0.327$ )]. Entretanto, verificou-se correlação moderada-baixa entre BNP D5 e BNP D1 [Figura 8, coeficiente de correlação $R=0.487(p<0.001)$ ] e alta correlação entre Delta-BNP e BNP D5 [Figura 9, coeficiente de correlação $R=0.893(p<0.001)$ ] 


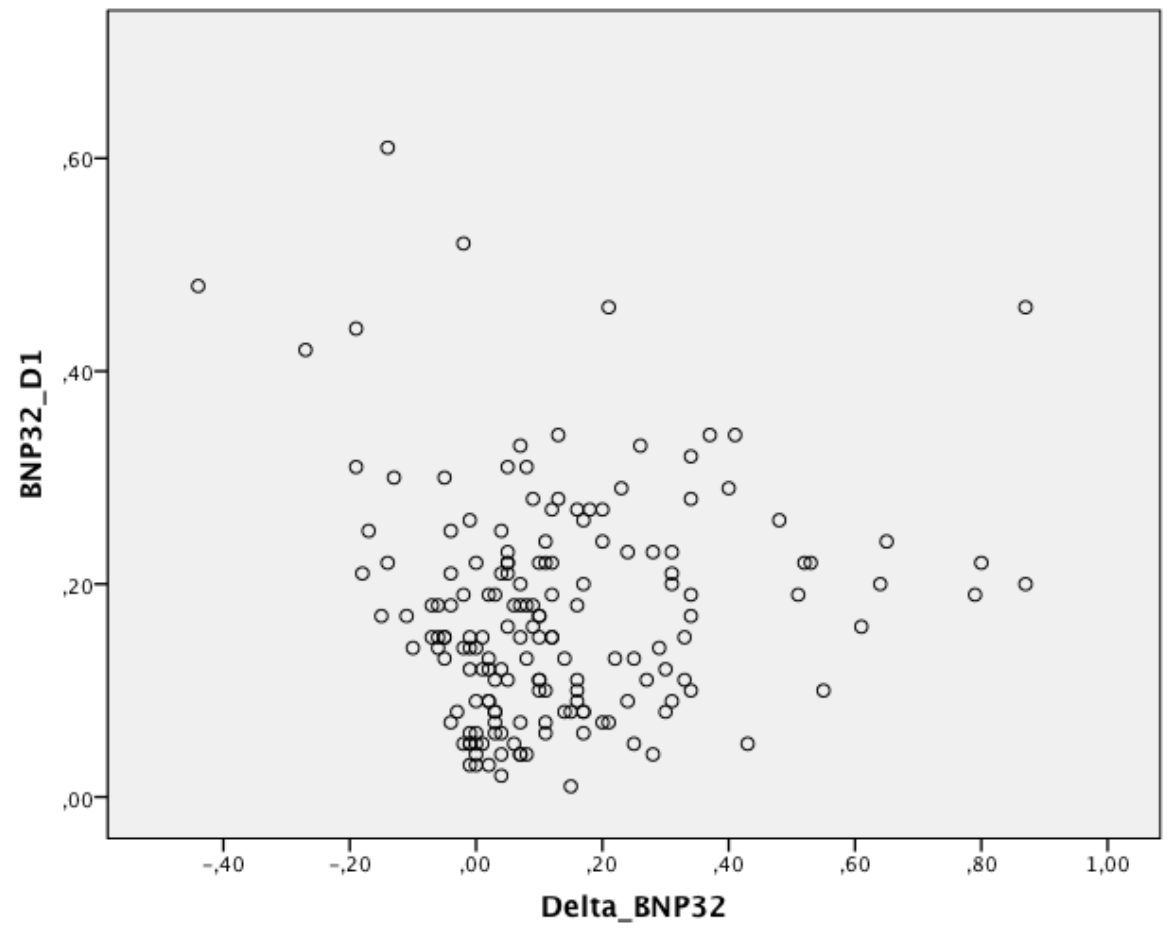

Figura 7: Regressão Linear // Delta-BNP x BNP D1 - $R=0.035(p=0.327)$

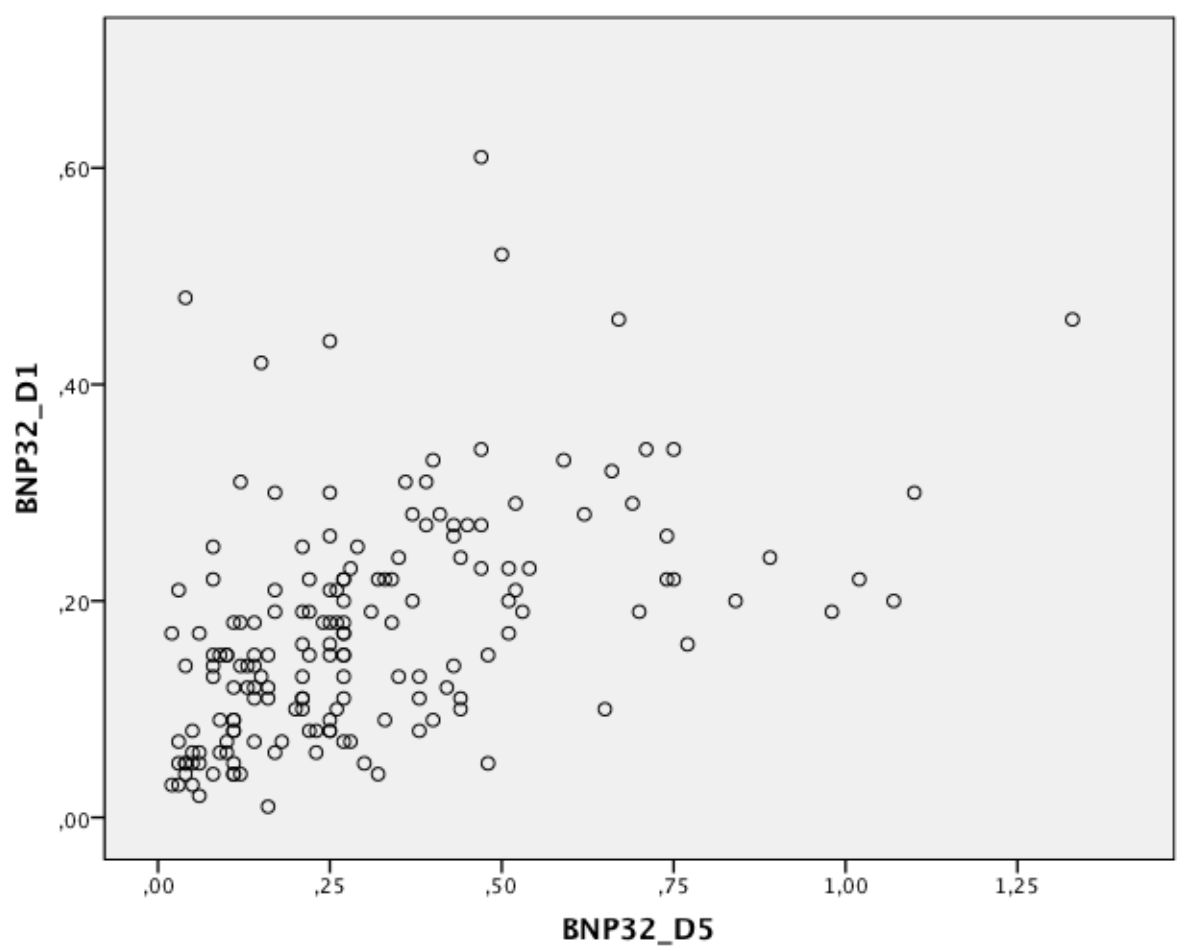

Figura 8: Regressão linear // BNP D5 x BNP D1 - $R=0.487(p<0.001)$ 


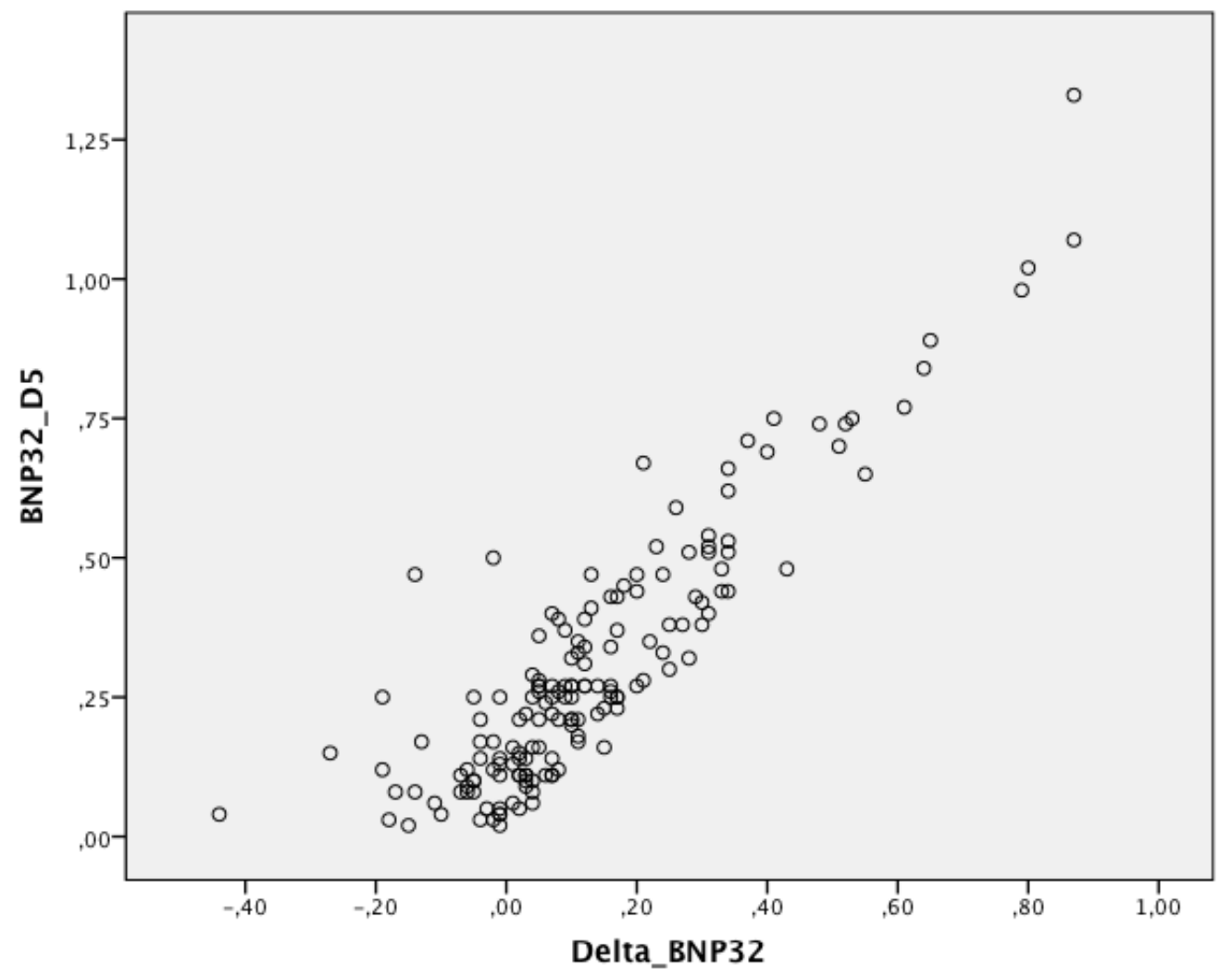

Figura 9: Regressão linear // Delta-BNP x BNP D5 - R=0.893 ( $p<0.001)$

Com o intuito de avaliar em termos de predição de risco o valor da adição do Delta-BNP ao escore GRACE, foi construída uma curva ROC (Figura 10). 


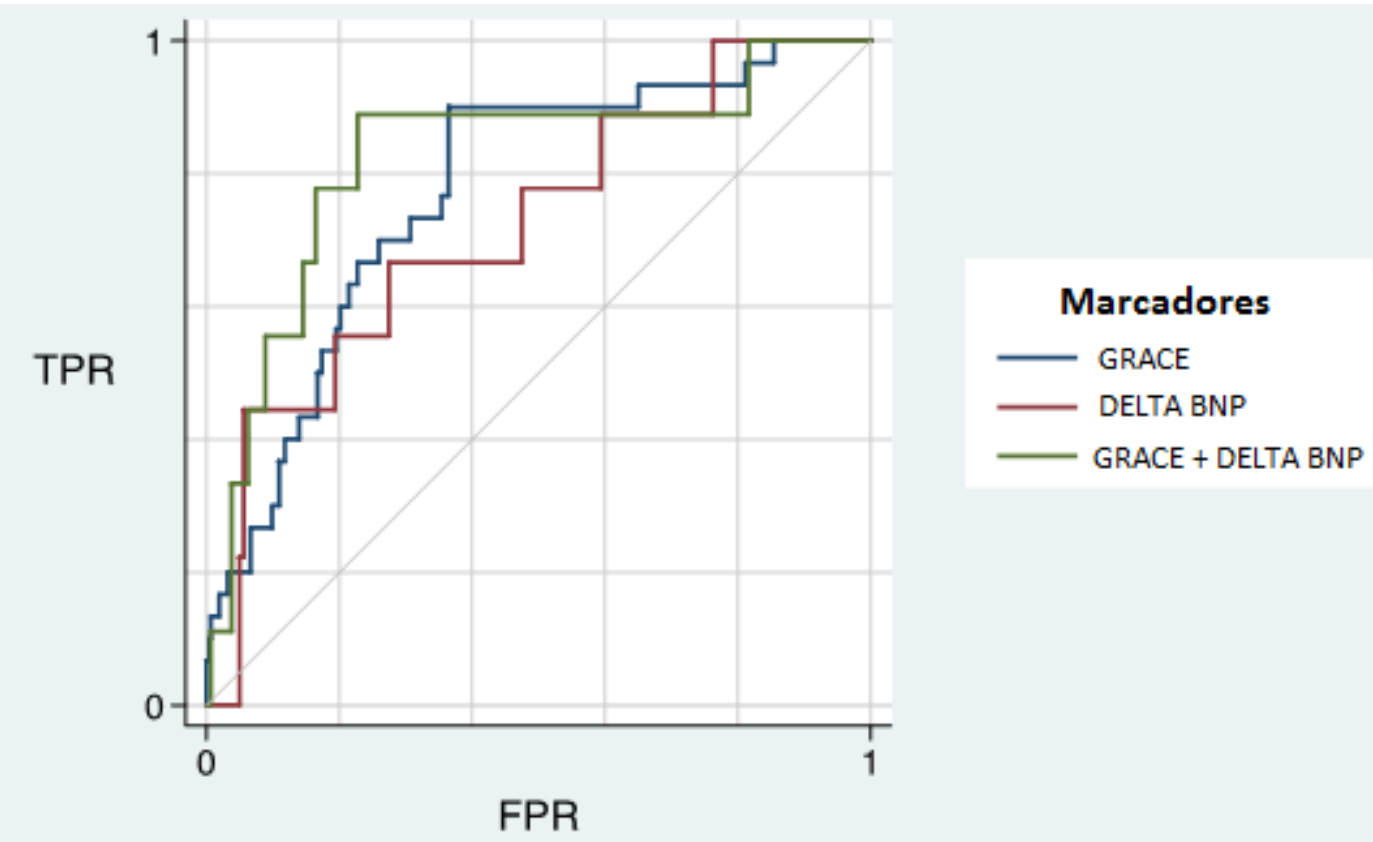

Figura 10: Curva ROC para discriminação de Morte Súbita e IAM fatal/não fatal em 30 dias. A área sob a curva (AUC) para o modelo combinado do escore GRACE + DeltaBNP [0.823 (IC 95\% 0.67-0.98; $p=0.001$ )] foi significativamente maior quando comparada às AUCs para o escore GRACE isolado [0.717 $(95 \% \mathrm{Cl}$ 0.53-0.90; $\mathrm{p}=0.029)$ ] ou para o Delta-BNP [0.731 (95\% Cl 0.56-0.90; $\mathrm{p}=0.020)]$. TPR: True positive rate (Taxa de Verdadeiros Positivos); FPR: false positive rate (Taxa de Falsos Positivos).

As áreas sob as curvas (AUC) foram 0,717 (IC 95\% 0.53 - 0.90) para o escore GRACE isoladamente e 0,823 (IC 95\% 0,67 - 0,98) para a combinação entre escore GRACE e BNP. (Tabela 55) O aumento de $14,78 \%$ observado na AUC foi estatisticamente significativo $(p=0.046)$ comparando-se as curvas $R O C$ através do comando comproc do programa STATA. O escore GRACE isolado apresentou uma estatística $C$ moderada de 0.709 ( $p=0.029$ ), porém a adição do Delta-BNP melhorou a discriminação de risco (Estatística $C=0.831, p=0.001$ ) 
Tabela 5 - Comparação dos modelos utilizando o escore GRACE isoladamente versus o escore GRACE associado ao Delta-BNP (GRACE + Delta-BNP) para discriminação de Morte Súbita Cardíaca e IAM fatal/não-fatal

Morte súbita e IAM fatal/não-fatal

GRACE $\quad$ GRACE + Delta-BNP $p$

Discriminação

Estatística C

$0.709(0.55-0.87)$

$0.831(0.68-0.97)$

0.042

Área sob a curva (AUC)

$0.717(0.53-0.90)$

$0.823(0.67-0.98)$

0.046

IDI Absoluto

$0.0198(p=0.069)$

cfNRI

Estatística índice (IC 95\%) $0.334(0.08-0.61 ; p=0.034)$

Calibração

Teste da razão de

verossimilhança, valor de $p$

0.008

Hosmer-Lemeshow $x^{2}$

$7.31(p=0.503)$

$4.83(p=0.776)$

Critério de informação

Bayesiano 
Com o objetivo de avaliar melhor o ganho obtido através da adição do DeltaBNP ao escore GRACE para predição de eventos em 30 dias pós-IAMCSST, realizouse a análise do índice de reclassificação líquida livre de categorias (cfNRI). O cfNRI confirmou significativa melhora de 33.4\% (IC 95\%: 8-61\%) na predição individual de risco em comparação ao escore GRACE isolado $(p=0.034)$, com adequada calibração de acordo com o critério de informação Bayesiano e o teste da razão de verossimilhança. De modo geral, a combinação Delta-BNP + escore GRACE melhorou a reclassificação em $55.2 \%$ ( $p=0.0447)$. Avaliando-se isoladamente os pacientes de risco intermediário, verificou-se que a reclassificação foi melhorada em $62.5 \%$ $(p=0.0231)$. Não obstante, dos 32 pacientes do grupo "livre de eventos" classificados como de risco intermediário pelo escore GRACE isoladamente, 21 foram reclassificados para o grupo de baixo risco e 1 para o alto risco com a adição do DeltaBNP ao escore GRACE, ao passo que os 2 pacientes de risco intermediário do grupo "eventos" foram todos reclassificados para alto risco. (Tabela 66)

Avaliando-se a adição do Delta-BNP ao GRACE escore para predição de eventos em 12 meses, observou-se que não houve melhora na estimativa de risco (cfNRI $p=0.7$ ). Além disso, considerando-se apenas a primeira medida, o BNP no D1 não melhorou a reclassificação de risco em 30 dias (cfNRI $p=0.8$ ) ou em 12 meses (cfNRI $\mathrm{p}=0.7$ ) quando comparado ao escore GRACE. 
Tabela 6 - Tabela de Melhora de Reclassificação

GRACE + DELTA-BNP

RECLASSIFICAÇÃO

GRACE ISOLADO

Baixo Intermediário Alto Total $\begin{gathered}\text { Para risco Para risco mais } \\ \text { mais alto baixo }\end{gathered}$

\section{Eventos}

$\begin{array}{cllllll}\text { Baixo } & 1 & 3 & 0 & 4 & 3 & 0 \\ \text { Intermediário } & 0 & 0 & 2 & 2 & 2 & 0 \\ \text { Alto } & 0 & 1 & 2 & 3 & 0 & 1\end{array}$

\section{Livre de eventos}

$\begin{array}{ccccccc}\text { Baixo } & 102 & 9 & 1 & 112 & 10 & 0 \\ \text { Intermediário } & 21 & 10 & 1 & 32 & 1 & 21 \\ \text { Alto } & 2 & 5 & 7 & 14 & 0 & 7\end{array}$

\section{Geral}

$\begin{array}{ccccccc}\text { Eventos } & 1 & 4 & 4 & 9 & 5 & 1 \\ \text { Livre de eventos } & 125 & 24 & 9 & 158 & 11 & 28\end{array}$

\section{Reclassificacão}

Geral

$55.2 \%(p=0.0447)$

Risco Intermediário

$62.5 \%(p=0.0231)$ 


\section{DISCUSSÃO}

Este é o primeiro estudo a sugerir que as concentrações plasmáticas de BNP melhoram a predição de risco em pacientes sem evidências clínicas de falência cardíaca durante IAMCSST. Além disso, os resultados obtidos indicam que duas medidas de BNP (admissão e D5) podem fornecer valor preditivo adicional ao escore GRACE quando comparado a única medida na admissão hospitalar. Uma maior elevação nas concentrações séricas de BNP entre a admissão e o quinto dia pós-IAM pode traduzir um marcador precoce de piora da função ventricular esquerda nos primeiros 30 dias após o evento coronariano, podendo essa ser a causa subjacente que embasa o achado de maior incidência dos eventos adversos estudados (Morte súbita cardíaca e recorrência de IAM) no grupo de pacientes com maior Delta-BNP. Sendo assim, pode-se explicar o motivo de a variação do BNP ter capacidade de predizer os eventos cardiovasculares tanto de forma isolada como complementar ao escore GRACE.

O BNP tem se mostrado de forma consistente como um marcador prognóstico independente nas síndromes coronarianas agudas. Altas concentrações séricas de BNP já foram associadas a insuficiência cardíaca congestiva (ICC), incidência de IM e mortalidade em ambos curto e longo seguimento após SCAs (24, 25). Entretanto, observa-se grande heterogeneidade em relação ao tempo em que é realizada a mensuração do BNP após o início dos sintomas, variando de algumas horas a 4 dias $(13,15,18)$, o que pode explicar a grande variabilidade encontrada na magnitude do poder preditivo do BNP entre diversos estudos prévios.

Estima-se que o padrão bifásico de elevação do BNP, descrito anteriormente na "Introdução" deste trabalho, ocorra em cerca de metade dos pacientes internados com IAM. (17) Em nosso estudo, pudemos identificar e quantificar o padrão bifásico através da análise da diferença entre duas medidas de BNP (à admissão, nas primeiras $12 \mathrm{~h}$ pós-IM, e no D5). A prevalência encontrada deste padrão foi de $54 \%$, avaliando-se somente pacientes com IAMCSST classificados como Killip I à admissão hospitalar. A alta prevalência de elevação tardia do BNP, caracterizando um padrão bifásico, associada à sua importância prognóstica podem explicar a melhor performance do Delta-BNP em relação a uma única medida de BNP (Admissão hospitalar - D1) no que se refere à reclassificação de risco em 30 dias. 
Avaliando-se os gráficos de regressão linear, observa-se que houve uma alta correlação entre o Delta- BNP e o BNP no D5. Tal achado pode suscitar questionamento sobre o real benefício de se utilizar a variável independente DeltaBNP (composto por duas dosagens) ao invés de única dosagem no D5. Entretanto, a escolha da variável Delta-BNP foi realizada a priori, buscando-se refletir de forma simplificada o comportamento de elevação bifásico do BNP após o infarto do miocárdio, não tendo sido realizadas análises com o BNP D5 como variável independente.

A combinação BNP e escore GRACE resultou em melhora na capacidade de predição de risco nas síndromes coronarianas agudas na maioria dos estudos prévios, porém não em todos. Em 600 pacientes com síndromes coronarianas sem elevação de segmento ST (isto é, IAMSSST e AI), as concentrações séricas de BNP medidas entre 24 e 96h após o início dos sintomas melhoraram a estatística-C de Harrell quando adicionadas ao escore GRACE (18). Em dois outros estudos, totalizando-se 1272 pacientes com IAMCSST (cerca de 10 a 25\% das coortes) e SCA sem elevação de segmento ST, foram encontrados resultados similares (19, 20). Além disso, mais recentemente, o BNP medido nas primeiras 24h após IAMCSST possibilitou melhora na capacidade preditiva do escore GRACE em 593 pacientes. (26) Em contraste, um estudo com 370 pacientes com SCA (sendo 50 pacientes com IAMCSST) não encontrou benefício adicional na combinação do BNP ao escore GRACE, apesar de relatar uma tendência de melhora da predição de morte em 1 ano (21). Faz-se necessário notar, entretanto, que todos os estudos citados avaliaram uma única medida de BNP, obtida em intervalos de tempo variados contados a partir do evento coronariano índice, não fazendo discernimento entre pacientes com ou sem sinais clínicos de insuficiência cardíaca à admissão hospitalar. Destarte, baseado nesta heterogeneidade observada, seria surpreendente se houvesse consistência nos achados destes estudos.

Elevadas concentrações plasmáticas de BNP são implicadas em remodelamento cardíaco adverso e maiores riscos de arritmias ventriculares e morte súbita cardíaca(27, 28). Além disso, foi aventada a hipótese por estudos experimentais de que os efeitos deletérios de altas concentrações de BNP possam se dever a uma ativação simpática paradoxal $(28,29)$. Possivelmente, a elevação do BNP em alguns pacientes ocorra por causa de uma atividade simpática exacerbada combinada ao remodelamento ventricular esquerdo, fatores que não podem ser 
explicados pelo escore GRACE em pacientes de baixo risco à admissão hospitalar após o IAMCSST e com pequena massa infartada. Isto ocorre porque provavelmente o escore GRACE e o Delta-BNP refletem atributos de risco distintos na predição de prognóstico adverso nos pacientes com IAMCSST e, portanto, o seu uso simultâneo pode melhorar a estratificação de risco.

Os métodos estatísticos de reclassificação líquida, NRI (Net Reclassification Improvement) e IDI (Integrated Discrimination Improvement), são maneiras relativamente recentes para avaliar a integração de novas informações a um modelo de predição de risco. Por serem ferramentas de desenvolvimento recente, existe alguma desconfiança em relação aos referidos testes, especialmente quando utilizados de forma isolada e sem uma calibração adequada. $(30,31)$ Neste estudo, evitamos estas "armadilhas" realizando outras análises simultâneas comparando as performances do escore GRACE e do GRACE + BNP através das áreas sob as curvas (AUC), estatística $\mathrm{C}$ de Harrell, múltiplos modelos de calibração como o teste de Hosmer-Lemeshow ( $p=0.5031$, boa adequação), o Critério de Informação Bayesiano $(B I C=2.951$, sugerindo uma melhoria na predição de risco) e o teste da razão de verossimilhança $(p=0.008)$. Desta forma, o modelo desenvolvido parece estar adequadamente calibrado e, portanto, menos vulnerável aos problemas dos métodos de reclassificação líquida utilizados (NRI e IDI).

A modelagem por splines é uma ferramenta estatística muito útil para avaliar a relação entre exposição e resposta, podendo ser aplicada à pesquisa clínica para análise de dados de sobrevivência e obtendo resultado mais preciso do que do modelo de Cox tradicional.(32) Algumas vantagens deste modelo incluem a possibilidade de detectar padrões relevantes de distribuição dos dados que podem ser negligenciados em análises de regressão mais simples, graças à potencial presença de diversos efeitos não-lineares inerentes ao campo da pesquisa clínica. Não obstante, considerase que a modelagem por splines possui um sofisticado refinamento matemático capaz de traduzir qualquer efeito não-linear de uma amostra de maneira bastante precisa. (33)

Uma das limitações deste estudo reside na pequena quantidade de pacientes arrolados. Embora o tamanho da amostra não seja o usual para a avaliação de um biomarcador, o poder do teste (1- $\beta$ ) atingiu 0,86 e foi precedido por um estudo piloto com 100 pacientes que orientou a escolha do tamanho amostral final. Entretanto, por conta dos amplos intervalos de confiança observados, deve-se considerar que a 
verdadeira magnitude dos efeitos não pôde ser definida com precisão. Mesmo assim, os achados encontrados merecem crédito pela consistência dos resultados entre os diversos modelos de reclassificação e discriminação de risco utilizados e seus respectivos métodos de calibração.

Um aspecto a ser considerado é o de que todos os pacientes avaliados tinham a bem definida entidade IAMCSST, e nenhum deles possuía sinais clínicos de insuficiência cardíaca à admissão hospitalar. Embora isto aumente a validade interna deste estudo, tal fato pode limitar a aplicabilidade dos achados observados a pacientes com características semelhantes, podendo não necessariamente ser adequado a outros contextos como o das síndromes coronarianas sem elevação de segmento ST ou mesmo o do IAMCSST nos indivíduos com sinais de falência de bomba, isto é, com classificação de Killip de II a IV. Além disso, a maior parte dos pacientes foi submetida a trombólise química $(74 \%$ e $79 \%$ nos grupos com BNP abaixo e acima da mediana, respectivamente), e os resultados encontrados podem não ser generalizáveis aos pacientes submetidos a angioplastia percutânea primária. 


\section{CONCLUSÃO}

A alta concentração plasmática de BNP nas primeiras $12 \mathrm{~h}$ pós-IAM e a elevação deste marcador nos dias subsequentes ao evento índice foram, ambas, associadas a pior desfecho prognóstico no curto prazo (30 dias pós-IAM) em pacientes com IAMCSST Killip I. O Delta-BNP melhorou a reclassificação de risco no curto prazo quando comparado tanto ao escore GRACE isolado ou ao GRACE + BNP admissional (D1). A incorporação destes achados é capaz de melhorar a precisão do escore GRACE em predizer eventos adversos, particularmente para indivíduos inicialmente considerados de baixo ou moderado risco. Propicia-se assim, estratificação clínica mais precisa, corroborando para escolha do tratamento mais adequado e condizente com o risco ao qual o paciente está exposto, no intuito de prevenir futuros desfechos desfavoráveis. 


\section{REFERÊNCIAS}

1. WHO WHO-. Fact Sheet - Cardiovascular diseases (CVDs) 2016 [cited 2016 01/Jun/2016]. Available from: http://www.who.int/mediacentre/factsheets/fs317/en/.

2. DATASUS. TABNET - Informações em Saúde - Estatísticas Vitais [cited 2016 01/Jun/2016]. Available from: http://tabnet.datasus.gov.br/cgi/deftohtm.exe?sim/cnv/obt10uf.def.

3. O'Gara PT, Kushner FG, Ascheim DD, Casey DE, Chung MK, de Lemos JA, et al. 2013 ACCF/AHA Guideline for the Management of ST-Elevation Myocardial InfarctionA Report of the American College of Cardiology Foundation/American Heart Association Task Force on Practice Guidelines. Journal of the American College of Cardiology. 2013;61(4):e78-e140.

4. GRACE Study Website. Available from: http://www.outcomes-umassmed.org/grace/. 5. Pieper KS, Gore JM, FitzGerald G, Granger CB, Goldberg RJ, Steg G, et al. Validity of a riskprediction tool for hospital mortality: the Global Registry of Acute Coronary Events. American heart journal. 2009;157(6):1097-105.

6. Granger CB, Goldberg RJ, Dabbous O, et al. PRedictors of hospital mortality in the global registry of acute coronary events. Archives of Internal Medicine. 2003;163(19):2345-53.

7. Elbarouni B, Goodman SG, Yan RT, Welsh RC, Kornder JM, Deyoung JP, et al. Validation of the Global Registry of Acute Coronary Event (GRACE) risk score for in-hospital mortality in patients with acute coronary syndrome in Canada. American heart journal. 2009;158(3):392-9.

8. Ramsay G, Podogrodzka M, McClure C, Fox KA. Risk prediction in patients presenting with suspected cardiac pain: the GRACE and TIMI risk scores versus clinical evaluation. QJM : monthly journal of the Association of Physicians. 2007;100(1):11-8.

9. Yan AT, Yan RT, Tan M, Casanova A, Labinaz M, Sridhar K, et al. Risk scores for risk stratification in acute coronary syndromes: useful but simpler is not necessarily better. European heart journal. 2007;28(9):1072-8.

10. Gale CP, Manda SO, Weston CF, Birkhead JS, Batin PD, Hall AS. Evaluation of risk scores for risk stratification of acute coronary syndromes in the Myocardial Infarction National Audit Project

(MINAP) database. Heart. 2009;95(3):221-7.

11. Mello BH, Oliveira GB, Ramos RF, Lopes BB, Barros CB, Carvalho Ede O, et al. Validation of the Killip-Kimball classification and late mortality after acute myocardial infarction. Arquivos brasileiros de cardiologia. 2014;103(2):107-17.

12. D'Ascenzo F, Biondi-Zoccai G, Moretti C, Bollati M, Omede P, Sciuto F, et al. TIMI, GRACE and alternative risk scores in Acute Coronary Syndromes: a meta-analysis of 40 derivation studies on 216,552 patients and of 42 validation studies on 31,625 patients. Contemporary clinical trials. 2012;33(3):507-14.

13. de Lemos JA, Morrow DA, Bentley JH, Omland T, Sabatine MS, McCabe CH, et al. The prognostic value of $\mathrm{B}$-type natriuretic peptide in patients with acute coronary syndromes. The New England journal of medicine. 2001;345(14):1014-21.

14. Omland T, Aakvaag A, Bonarjee VV, Caidahl K, Lie RT, Nilsen DW, et al. Plasma brain natriuretic peptide as an indicator of left ventricular systolic function and long-term survival after acute myocardial infarction. Comparison with plasma atrial natriuretic peptide and $\mathrm{N}$-terminal proatrial natriuretic peptide. Circulation. 1996;93(11):1963-9.

15. Richards AM, Nicholls MG, Yandle TG, Ikram H, Espiner EA, Turner JG, et al. Neuroendocrine prediction of left ventricular function and heart failure after acute myocardial infarction. The

Christchurch Cardioendocrine Research Group. Heart. 1999;81(2):114-20.

16. Mega JL, Morrow DA, De Lemos JA, Sabatine MS, Murphy SA, Rifai N, et al. B-type natriuretic peptide at presentation and prognosis in patients with ST-segment elevation myocardial infarction: an ENTIRE-TIMI-23 substudy. Journal of the American College of Cardiology. 2004;44(2):335-9. 
17. Morita E, Yasue $H$, Yoshimura M, Ogawa $H$, Jougasaki M, Matsumura $T$, et al. Increased plasma levels of brain natriuretic peptide in patients with acute myocardial infarction. Circulation. 1993;88(1):82-91.

18. García-Alvarez A, Regueiro A, Hernández J, Kasa G, Sitges M, Bosch X, et al. Additional value of B-type natriuretic peptide on discrimination of patients at risk for mortality after a non-STsegment elevation acute coronary syndrome. European Heart Journal: Acute Cardiovascular Care. 2014;3(2):132-40.

19. Guidez T, Maréchaux S, Pinçon C, Lamour H, Barrailler S, Decourcelle V, et al. Addition of Btype natriuretic peptide to the GRACE score to predict outcome in acute coronary syndrome: a retrospective (development) and prospective (validation) cohort-based study. Emergency Medicine Journal. 2012;29(4):274-9.

20. Ang DSC, Wei L, Kao MPC, Lang CC, Struthers AD. A comparison between B-type natriuretic peptide, Global Registry of Acute Coronary Events (GRACE) score and their combination in ACS risk stratification. Heart. 2009;95(22):1836-42.

21. Meune C, Drexler B, Haaf P, Reichlin T, Reiter M, Meissner J, et al. The GRACE score's performance in predicting in-hospital and 1-year outcome in the era of high-sensitivity cardiac troponin assays and B-type natriuretic peptide. Heart. 2011;97(18):1479-83.

22. Killip T, 3rd, Kimball JT. Treatment of myocardial infarction in a coronary care unit. A two year experience with 250 patients. The American journal of cardiology. 1967;20(4):457-64.

23. Lang RM, Badano LP, Mor-Avi V, Afilalo J, Armstrong A, Ernande L, et al. Recommendations for cardiac chamber quantification by echocardiography in adults: an update from the American Society of Echocardiography and the European Association of Cardiovascular Imaging. European heart journal cardiovascular Imaging. 2015;16(3):233-70.

24. Omland T, Persson A, Ng L, O'Brien R, Karlsson T, Herlitz J, et al. N-terminal pro-B-type natriuretic peptide and long-term mortality in acute coronary syndromes. Circulation. 2002;106(23):2913-8.

25. Grabowski M, Filipiak KJ, Malek LA, Karpinski G, Huczek Z, Stolarz P, et al. Admission B-type natriuretic peptide assessment improves early risk stratification by Killip classes and TIMI risk score in patients with acute ST elevation myocardial infarction treated with primary angioplasty. International journal of cardiology. 2007;115(3):386-90.

26. Parenica J, Kala P, Pavkova MG, Tomandl J, Spinar J, Littnerova S, et al. Natriuretic peptides, nitrite/nitrate and superoxide dismutase have additional value on top of the GRACE score in prediction of one-year mortality and rehospitalisation for heart failure in STEMI patients - Multiple biomarkers prospective cohort study. International journal of cardiology. 2016;211:96-104.

27. Scott PA, Barry J, Roberts PR, Morgan JM. Brain natriuretic peptide for the prediction of sudden cardiac death and ventricular arrhythmias: a meta-analysis. European journal of heart failure. 2009;11(10):958-66.

28. Thireau J, Karam S, Fauconnier J, Roberge S, Cassan C, Cazorla O, et al. Functional evidence for an active role of B-type natriuretic peptide in cardiac remodelling and pro-arrhythmogenicity. Cardiovascular research. 2012;95(1):59-68.

29. Chan NY, Seyedi N, Takano K, Levi R. An unsuspected property of natriuretic peptides: promotion of calcium-dependent catecholamine release via protein kinase G-mediated phosphodiesterase type 3 inhibition. Circulation. 2012;125(2):298-307.

30. Hilden J, Gerds TA. A note on the evaluation of novel biomarkers: do not rely on integrated discrimination improvement and net reclassification index. Statistics in medicine. 2014;33(19):340514.

31. Pepe MS, Janes $\mathrm{H}, \mathrm{Li} \mathrm{Cl}$. Net risk reclassification $\mathrm{p}$ values: valid or misleading? Journal of the National Cancer Institute. 2014;106(4):dju041.

32. Hastie T, Sleeper L, Tibshirani R. Flexible covariate effects in the proportional hazards model. Breast cancer research and treatment. 1992;22(3):241-50.

33. Cleophas TJ. Clinical Trials: Spline Modeling is Wonderful for Nonlinear Effects. American journal of therapeutics. 2016;23(3):e844-9. 



\section{ANEXO A - PARECER FAVORÁVEL DO COMITÊ DE ÉTICA EM PESQUISA DA SECRETARIA DE SAÚDE DO DISTRITO FEDERAL}

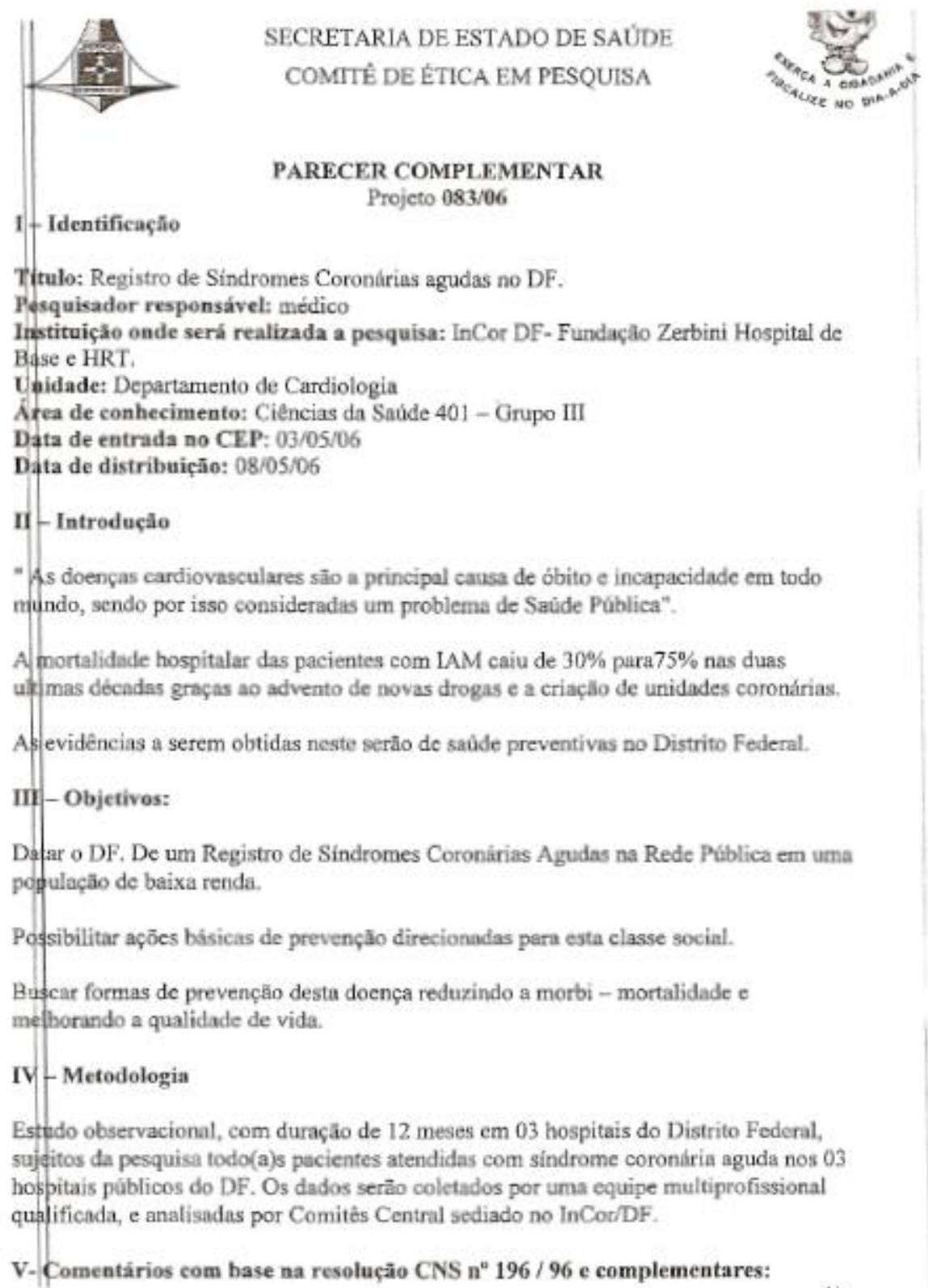

Observa-se que o mesmo contempla as normas estabelecidas quanto aos documentos de instruçâo do Protocola de Pesquisa, contudo, falta anexar os termos de concordâneia dos diretores do Hospital de Base e do HRT. (PENDENCIA ATENDIDA).

VI - Conclusão - Projeto aprovado.

Brasillia, 28 de maio de 2006

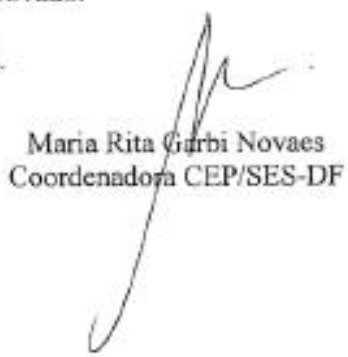

